JOURNAL OF THE

AMERICAN MATHEMATICAL SOCIETY

Volume 22, Number 3, July 2009, Pages 771-796

S 0894-0347(08)00601-2

Article electronically published on April 25, 2008

\title{
BOUNDARY STRUCTURE AND SIZE IN TERMS OF INTERIOR AND EXTERIOR HARMONIC MEASURES IN HIGHER DIMENSIONS
}

\author{
C. KENIG, D. PREISS, AND T. TORO
}

\section{INTRODUCTION}

In this work we introduce the use of powerful tools from geometric measure theory (GMT) to study problems related to the size and structure of sets of mutual absolute continuity for the harmonic measure $\omega^{+}$of a domain $\Omega=\Omega^{+} \subset \mathbb{R}^{n}$ and the harmonic measure $\omega^{-}$of $\Omega^{-}, \Omega^{-}=\operatorname{int}\left(\Omega^{c}\right)$ in dimension $n \geq 3$. These tools come mainly from Preiss' work (see [19]), in which he proved that if the $m$-density of a Radon measure $\mu$ in $\mathbb{R}^{n}$ exists and is positive and finite, for $\mu$-almost every point of $\mathbb{R}^{n}$, then $\mu$ is $m$-rectifiable; see [18. for all the relevant definitions. These techniques are combined with the blow-up analysis developed by Kenig-Toro [14], the properties of harmonic functions on non-tangentially accessible (NTA) domains [11] and the monotonicity formula of Alt-Caffarelli-Friedman [1] to obtain analogs for $n \geq 3$ of some well-known results when $n=2$.

Let us first briefly describe some of the 2-dimensional results. Thus, let $\Omega \subset$ $\mathbb{R}^{2}$ be a simply-connected domain, bounded by a Jordan curve and let $\omega$ be the harmonic measure associated to $\Omega$ (see 9]). Then we can write $\partial \Omega$ as a disjoint union, with the following properties:

$$
\partial \Omega=G \cup S \cup N
$$

i) $\omega(N)=0$.

ii) In $G, \omega \ll \mathcal{H}^{1} \ll \omega$, where $\mathcal{H}^{s}$ denotes an $s$-dimensional Hausdorff measure.

iii) Every point of $G$ is the vertex of a cone in $\Omega$. Moreover if $C$ denotes the set of "cone points" of $\partial \Omega$, then $\mathcal{H}^{1}(C \backslash G)=0$ and $\omega(C \backslash G)=0$.

iv) $\mathcal{H}^{1}(S)=0$.

v) $S$ consists ( $\omega$ a.e.) of "twist points" (a geometrical characterization of $S$ ). See [9] for the definition of a twist point.

vi) For $\omega$ a.e. $Q \in G$ we have that

$$
\lim _{r \rightarrow 0} \frac{\omega(B(Q, r) \cap \partial \Omega)}{r}=L \text { exists and } \quad 0<L<\infty .
$$

Received by the editors October 25, 2007.

2000 Mathematics Subject Classification. Primary 28A33, 31A15.

The first author was partially supported by NSF grant DMS-0456583.

The third author was partially supported by NSF grant DMS-0600915.

(C)2008 American Mathematical Society Reverts to public domain 28 years from publication 
vii) At $\omega$ a.e. point $Q \in S$ we have

$$
\begin{aligned}
& \limsup _{r \rightarrow 0} \frac{\omega(B(Q, r) \cap \partial \Omega)}{r}=+\infty, \\
& \liminf _{r \rightarrow 0} \frac{\omega(B(Q, r) \cap \partial \Omega)}{r}=0 .
\end{aligned}
$$

These results are a combination of work of Makarov, McMillan, Pommerenke and Choi. See [9] for the precise references.

Recall that the Hausdorff dimension of $\omega$ (denote by $\mathcal{H}-\operatorname{dim} \omega$ ) is defined by

$$
\begin{aligned}
\mathcal{H}-\operatorname{dim} \omega= & \inf \left\{k: \text { there exists } E \subset \partial \Omega \text { with } \mathcal{H}^{k}(E)=0\right. \text { and } \\
& \left.\omega(E \cap K)=\omega(\partial \Omega \cap K) \text { for all compact sets } K \subset \mathbb{R}^{n}\right\} .
\end{aligned}
$$

Important work of Makarov [17] shows that for simply-connected domains in $\mathbb{R}^{2}$, $\mathcal{H}-\operatorname{dim} \omega=1$, establishing Oksendal's conjecture in dimension 2. Carleson [6, and Jones and Wolff 12 proved that, in general, for domains in $\mathbb{R}^{2}$ with a well-defined harmonic measure $\omega, \mathcal{H}-\operatorname{dim} \omega \leq 1$. T. Wolff 21 showed, by a deep example, that for $n \geq 3$, Oksendal's conjecture $(\mathcal{H}-\operatorname{dim} \omega=n-1)$ fails. He constructed what we will call "Wolff snowflakes", domains in $\mathbb{R}^{3}$ for which $\mathcal{H}-\operatorname{dim} \omega>2$ and others for which $\mathcal{H}-\operatorname{dim} \omega<2$. In Wolff's construction, the domains have a certain weak regularity property: they are non-tangentially accessible domains (NTA) in the sense of [11. In fact, they are 2-sided NTA domains (i.e. $\Omega$ and $\operatorname{int}\left(\Omega^{c}\right)$ are both NTA), and this plays an important role in his estimates. Here, whenever we refer to a "Wolff snowflake," we will mean a 2 -sided NTA domain in $\mathbb{R}^{n}$, for which $\mathcal{H}-\operatorname{dim} \omega \neq n-1$. In [16, Lewis, Verchota and Vogel reexamined Wolff's construction and were able to produce "Wolff snowflakes" in $\mathbb{R}^{n}, n \geq 3$, for which $\mathcal{H}-\operatorname{dim} \omega^{ \pm}>n-1$, and others for which $\mathcal{H}-\operatorname{dim} \omega^{ \pm}<n-1$. They also observed, as a consequence of the monotonicity formula in [1], that if $\omega^{+} \ll \omega^{-} \ll \omega^{+}$, then $\mathcal{H}-\operatorname{dim} \omega^{ \pm} \geq n-1$.

Returning to the case of $n=2$, when $\Omega$ is again simply connected, bounded by a Jordan curve, $\omega^{+}=\omega$ and $\omega^{-}$equals the harmonic measure for $\operatorname{int}\left(\Omega^{c}\right)$, Bishop, Carleson, Garnett and Jones [4 showed that, if $E \subset \partial \Omega, \omega^{+}(E)>0, \omega^{-}(E)>0$, then $\omega^{+} \perp \omega^{-}$on $E$ if and only if $\mathcal{H}^{1}(\operatorname{Tn}(\partial \Omega) \cap E)=0$, where $Q \in \operatorname{Tn}(\partial \Omega) \subset \partial \Omega$ if $\partial \Omega$ has a unique tangent line at $Q$. Recall that $\partial \Omega$ admits a decomposition relative to $\omega^{ \pm}, \partial \Omega=G^{ \pm} \cup S^{ \pm} \cup N^{ \pm}$(see (1.1)). Let $E \subset \partial \Omega$ be such that $\omega^{+} \ll \omega^{-} \ll \omega^{+}$on $E$ and $\omega^{ \pm}(E)>0$; then, because of [4, modulo sets of $\omega^{ \pm}$ measure 0, $E \subset \operatorname{Tn}(\partial \Omega)$. Using Beurling's inequality, i.e. the fact that for $Q \in \partial \Omega$ and $r>0, \omega^{+}(B(Q, r)) \omega^{-}(B(Q, r)) \leq C r^{2}$, and the characterization above for $G^{ \pm}$ and $S^{ \pm}$(see ii), vi) and vii)) we conclude that $\omega^{+} \ll \mathcal{H}^{1} \ll \omega^{-} \ll \omega^{+}$on $E$. Thus, sets of mutual absolute continuity of $\omega^{-}, \omega^{+}$are "regular" and hence obviously of dimension 1 .

In 3, motivated by this last result, Bishop asked whether in the case of $\mathbb{R}^{n}, n \geq$ 3 , if $\omega^{-}, \omega^{+}$are mutually absolutely continuous on a set $E \subset \partial \Omega, \omega^{ \pm}(E)>0$, then $\omega^{ \pm}$are mutually absolutely continuous with respect to $\mathcal{H}^{n-1}$ on $E$ (modulo a set of $\omega^{ \pm}$measure zero) and hence $\operatorname{dim}_{\mathcal{H}}(E)=n-1$. On the other hand, Lewis, Verchota and Vogel [16] conjectured that there are "Wolff snowflakes" in $\mathbb{R}^{n}, n \geq 3$, with $\mathcal{H}-\operatorname{dim} \omega^{ \pm}>n-1$, for which $\omega^{+}, \omega^{-}$are not mutually singular. In this paper we study these issues for domains which verify the weak regularity hypothesis of being 2-sided locally NTA (a condition which, of course, Wolff snowflakes verify). This condition ensures that we have scale invariant estimates for harmonic measures. In 
the $n=2$ case, the condition is equivalent to locally being a quasi-circle, but it is weaker than that when $n>2$. We expect that versions of our results will still be valid under even weaker regularity assumptions. We would like to stress though that no flatness assumption is made in this work; that has been one of the main points that we wanted to address here, as well as the introduction of the techniques from GMT ([19]), combined with the blow-up analysis in [14].

Our main result is that, for $n \geq 3, \partial \Omega=\Gamma^{*} \cup S \cup N$, where $\omega^{+} \perp \omega^{-}$on $S$, $\omega^{ \pm}(N)=0$, and on $\Gamma^{*}, \omega^{+} \ll \omega^{-} \ll \omega^{+}, \operatorname{dim}_{\mathcal{H}} \Gamma^{*} \leq n-1$, and if $\omega^{ \pm}\left(\Gamma^{*}\right)>0$, $\operatorname{dim}_{\mathcal{H}} \Gamma^{*}=n-1$, where $\operatorname{dim}_{\mathcal{H}}$ denotes the Hausdorff dimension of a set. As a consequence there can be no "Wolff snowflake" for which $\omega^{+}, \omega^{-}$are mutually absolutely continuous. We also show that $\Gamma^{*}=\Gamma_{g}^{*} \cup \Gamma_{b}^{*} \cup Z, \omega^{ \pm}(Z)=0$, where in $\Gamma_{g}^{*}, \mathcal{H}^{n-1}$ is $\sigma$-finite, $\omega^{-} \ll \mathcal{H}^{n-1} \ll \omega^{+} \ll \omega^{-}$, while on $\Gamma_{b}^{*}$, for a Borel set $E$ we have that if $\omega^{ \pm}\left(\Gamma_{b}^{*} \cap E\right)>0$, then $\mathcal{H}^{n-1}\left(\Gamma_{b}^{*} \cap E\right)=+\infty$. If we further assume that $\mathcal{H}^{n-1}\left\llcorner\partial \Omega\right.$ is a Radon measure, then we show that $\Gamma^{*}$ is $(n-1)$ rectifiable. In this case, we must have $\omega^{ \pm}\left(\Gamma_{b}^{*}\right)=0$, and hence $\partial \Omega=\Gamma_{g}^{*} \cup S \cup \widetilde{N}, \omega^{ \pm}(\widetilde{N})=0$ and $\Gamma_{g}^{*}$ is rectifiable.

Our approach is the following. Using the blow-up analysis developed in [14, at $\omega^{ \pm}$a.e. point on the set where $\omega^{+}$and $\omega^{-}$are mutually absolutely continuous, the tangent measures to $\omega^{ \pm}$(in the sense of [19], [18]) are harmonic measures associated to the zero set of a harmonic polynomial (see Theorem 3.4). Using the fact that for almost every point a tangent measure to a tangent measure is a tangent measure, (see [18) and the fact that the zero set of a harmonic polynomial is smooth except for a set of Hausdorff dimension $n-2$ (see [10]), one shows that at $\omega^{ \pm}$a.e. point on this set, $(n-1)$ flat measures always arise as tangent measures to $\omega^{ \pm}$. They correspond to linear harmonic polynomials. We then show, and this is the crucial step, that if one tangent measure is flat, on the set of mutual absolute continuity, then all tangent measures are flat (see Theorem 4.1). To accomplish this we use a connectivity argument from [19]. The key point is that if a tangent measure is not flat, being the harmonic measure associated to the zero set of a harmonic polynomial of degree higher than 1, its tangent measure at infinity is far from flat (see Lemma 4.1), and a connectivity argument in $d$-cones of measures, in the metric introduced by Preiss in [19], gives a contradiction. Modulo a set of $\omega^{ \pm}$measure 0 , let $\Gamma^{*}$ be the points in the set of mutual absolute continuity for which one (and hence all) tangent measures are $(n-1)$ flat. An easy argument (see Lemma 2.4 and the proof of Theorem 4.1) shows that $\operatorname{dim}_{\mathcal{H}} \Gamma^{*} \leq n-1$. To conclude that if $\omega^{ \pm}\left(\Gamma^{*}\right)>0$, then $\operatorname{dim}_{\mathcal{H}} \Gamma^{*}=n-1$, one uses the Alt-Caffarelli-Friedman monotonicity formula of [1] as in [16. If $\mathcal{H}^{n-1}\left\llcorner\partial \Omega\right.$ is a Radon measure, one can show that its density on $\Gamma^{*}$ is $1, \mathcal{H}^{n-1}\left\llcorner\partial \Omega\right.$ a.e., which shows that $\Gamma^{*}$ is $(n-1)$ rectifiable (see [18).

The results in Theorem 2.1 and Corollary 2.1 are very general. We believe they should be useful in other situations where questions of size and structure of the support of a measure arise.

\section{Some ReSults in GeOMEtric meAsure theOry}

We start this section with some basic definitions in GMT. Then we recall two families of "distances" between Radon measures in Euclidean space which are compatible with weak convergence. They were initially introduced in [19]. We finish 
the section with a general theorem which can be used to derive geometric properties of two domains' common boundary from comparisons between their harmonic measures.

Recall that if $\Phi$ is a Radon measure in $\mathbb{R}^{n}$, then

$$
\text { spt } \Phi=\left\{x \in \mathbb{R}^{n}: \Phi(B(x, r))>0 \quad \forall r>0\right\} .
$$

Definition 2.1. Let $\Phi$ and $\Psi$ be Radon measures in $\mathbb{R}^{n}$. Let $K$ be a compact set in $\mathbb{R}^{n}$ and define

i) $F_{K}(\Phi)=\int \operatorname{dist}\left(z, K^{c}\right) d \Phi(z)$.

ii) If $F_{K}(\Phi)+F_{K}(\Psi)<\infty$, let

$$
F_{K}(\Phi, \Psi)=\sup \left\{\left|\int f d \Phi-\int f d \Psi\right|: \operatorname{spt} f \subset K, f \geq 0, \operatorname{Lip} f \leq 1\right\} .
$$

We denote by $F_{r}(\Phi)=F_{B(0, r)}(\Phi)$. Note that $F_{K}(\Phi)=F_{K}(\Phi, 0)$.

Remark 2.1. Let $\Phi$ be a Radon measure in $\mathbb{R}^{n}$. For $x \in \mathbb{R}^{n}$ and $r>0$ define $T_{x, r}: \mathbb{R}^{n} \rightarrow \mathbb{R}^{n}$ by the formula $T_{x, r}(z)=(z-x) / r$. Note that:

i) $T_{x, r}[\Phi](B(0, s)):=\Phi\left(T_{x, r}^{-1}(B(0, s))\right)=\Phi(B(x, s r))$ for every $s>0$.

ii) $\int f(z) d T_{x, r}[\Phi](z)=\int f\left(\frac{z-x}{r}\right) d \Phi(z)$ whenever at least one of these integrals is defined.

iii) $F_{B(x, r)}(\Phi)=r F_{1}\left(T_{x, r}[\Phi]\right)$.

iv) $F_{B(x, r)}(\Phi, \Psi)=r F_{1}\left(T_{x, r}[\Phi], T_{x, r}[\Psi]\right)$.

Definition 2.2. Let $\mu, \mu_{1}, \mu_{2}, \ldots$ be Radon measures on $\mathbb{R}^{n}$. We say that $\mu_{i} \rightarrow \mu$ or $\lim _{i \rightarrow \infty} \mu_{i}=\mu$ if

i) $\limsup _{i \rightarrow \infty} F_{K}\left(\mu_{i}\right)<\infty$ for every compact set $K \subset \mathbb{R}^{n}$.

ii) $\lim _{i \rightarrow \infty} F_{K}\left(\mu_{i}, \mu\right)=0$ for every compact set $K \subset \mathbb{R}^{n}$.

Definition 2.3. Let $\mu, \mu_{1}, \mu_{2}, \ldots$ be Radon measures on $\mathbb{R}^{n}$. We say that $\left\{\mu_{i}\right\}$ converges weakly to $\mu, \mu_{i} \rightarrow \mu$ if

$$
\lim _{r \rightarrow \infty} \int f d \mu:=\int f d \mu \quad \forall \phi \in C_{c}\left(\mathbb{R}^{n}\right) .
$$

Lemma 2.1 ([19], Proposition 1.11). Let $\mu_{1}, \mu_{2} \ldots$ and $\mu$ be Radon measures on $\mathbb{R}^{n}$ such that $\limsup _{i \rightarrow \infty} \mu_{i}(K)<\infty$ for each compact set $K$ in $\mathbb{R}^{n}$. Then $\mu_{i} \rightarrow \mu$ if and only if $\mu_{i} \rightarrow \mu$.

Lemma 2.2 ([18], Lemma 14.13). Let $\mu_{1}, \mu_{2}, \ldots$ and $\mu$ be Radon measures on $\mathbb{R}^{n}$. Then $\mu_{i} \rightarrow \mu$ if and only if

$$
\lim _{i \rightarrow \infty} F_{r}\left(\mu_{i}, \mu\right)=0 \quad \forall r>0 .
$$

We now introduce a scale invariant relative of $F_{r}$, which behaves well under weak convergence and scaling.

Definition $2.4([19, \S 2)$.

i) A set $\mathcal{M}$ of non-zero Radon measures in $\mathbb{R}^{n}$ will be called a cone if $c \Psi \in \mathcal{M}$ whenever $\Psi \in \mathcal{M}$ and $c>0$. 
ii) A cone $\mathcal{M}$ will be called a $d$-cone if $T_{0, r}[\Psi] \in \mathcal{M}$ whenever $\Psi \in \mathcal{M}$ and $r>0$.

iii) Let $\mathcal{M}$ be a $d$-cone and $\Phi$ a Radon measure in $\mathbb{R}^{n}$ such that for $s>0$, $0<F_{s}(\Phi)<\infty$, we define the distance between $\Phi$ and $\mathcal{M}$ by

$$
d_{s}(\Phi, \mathcal{M})=\inf \left\{F_{s}\left(\frac{\Phi}{F_{s}(\Phi)}, \Psi\right): \Psi \in \mathcal{M} \text { and } F_{s}(\Psi)=1\right\} .
$$

We also define

$$
d_{s}(\Phi, \mathcal{M})=1 \text { if } F_{s}(\Phi)=0 \text { or } F_{s}(\Phi)=+\infty .
$$

Remark 2.2. Note that if $\mathcal{M}$ is a $d$-cone and $\Phi$ is a Radon measure,

i) $d_{s}(\Phi, \mathcal{M}) \leq 1$,

ii) $d_{s}(\Phi, \mathcal{M})=d_{1}\left(T_{0, s}[\phi], \mathcal{M}\right)$,

iii) if $\mu=\lim _{i \rightarrow \infty} \mu_{i}$ and $F_{s}(\mu)>0$, then $d_{s}(\mu, \mathcal{M})=\lim _{i \rightarrow \infty} d_{s}\left(\mu_{i}, \mathcal{M}\right)$.

In fact if $\mu=\lim _{i \rightarrow \infty} \mu_{i}$, then by Lemma 2.1 $\mu_{i} \rightarrow \mu$ and for $s>0$

$$
F_{s}\left(\mu_{i}\right)=\int(s-|z|)^{+} d \mu_{i} \rightarrow \int(s-|z|)^{+} d \mu=F_{s}(\mu) .
$$

Thus without loss of generality we may assume that $F_{s}\left(\mu_{i}\right)>0$ (at least for $i$ large enough). Since $\lim _{i \rightarrow \infty} \mu_{i}=\mu$ then $\limsup F_{s}\left(\mu_{i}\right)<\infty$ and therefore $F_{s}(\mu)<\infty$. Let $\Psi \in \mathcal{M}$ such that $F_{s}(\Psi)=1$; then

$$
\begin{aligned}
F_{s}\left(\frac{\mu}{F_{s}(\mu)}, \Psi\right) \leq & F_{s}\left(\frac{\mu}{F_{s}(\mu)}, \frac{\mu_{i}}{F_{s}(\mu)}\right)+F_{s}\left(\frac{\mu_{i}}{F_{s}(\mu)}, \frac{\mu_{i}}{F_{s}\left(\mu_{i}\right)}\right) \\
& +F_{s}\left(\frac{\mu_{i}}{F_{s}\left(\mu_{i}\right)}, \Psi\right) \\
\leq & \frac{1}{F_{s}(\mu)} F_{s}\left(\mu, \mu_{i}\right)+F_{s}\left(\mu_{i}\right)\left|\frac{1}{F_{s}(\mu)}-\frac{1}{F_{s}\left(\mu_{i}\right)}\right| \\
& +F_{s}\left(\frac{\mu_{i}}{F_{s}\left(\mu_{i}\right)}, \Psi\right) .
\end{aligned}
$$

Thus for any $\Psi \in \mathcal{M}$ with $F_{s}(\Psi)=1$ we have

$$
d_{s}(\mu, \mathcal{M}) \leq \frac{1}{F_{s}(\mu)} F_{s}\left(\mu, \mu_{i}\right)+F_{s}(\mu)\left|\frac{1}{F_{s}(\mu)}-\frac{1}{F_{s}\left(\mu_{i}\right)}\right|+F_{s}\left(\frac{\mu}{F_{s}\left(\mu_{i}\right)}, \Psi\right)
$$

which implies

$$
d_{s}(\mu, \mathcal{M}) \leq \frac{F_{s}\left(\mu, \mu_{i}\right)}{F_{s}(\mu)}+F_{s}(\mu)\left|\frac{1}{F_{s}(\mu)}-\frac{1}{F_{s}\left(\mu_{i}\right)}\right|+d_{s}\left(\mu_{i}, \mathcal{M}\right) .
$$

Letting $i \rightarrow \infty$ and combining (2.4) and (2.7) we have that

$$
d_{s}(\mu, \mathcal{M}) \leq \liminf _{i \rightarrow \infty} d_{s}\left(\mu_{i}, \mathcal{M}\right) .
$$

A similar calculation done reversing the roles of $\mu$ and $\mu_{i}$ yields the inequality

$$
\limsup _{i \rightarrow \infty} d_{s}\left(\mu_{i}, \mathcal{M}\right) \leq d_{s}(\mu, \mathcal{M})
$$

which proves statement iii) in Remark 2.2 . 


\section{Definition 2.5.}

i) Let $\eta$ be a Radon measure in $\mathbb{R}^{n}$. Let $x \in \mathbb{R}^{n}$; a non-zero Radon measure $\nu$ in $\mathbb{R}^{n}$ is said to be a tangent measure of $\eta$ at $x$ if there are sequences $r_{k} \searrow 0$ and $c_{k}>0$ such that $\nu=\lim _{k \rightarrow \infty} c_{k} T_{x, r_{k}}[\eta]$.

ii) The set of all tangent measures to $\eta$ at $x$ is denoted by $\operatorname{Tan}(\eta, x)$.

Remark 2.3. For $\eta$ a non-zero Radon measure and $x \in \mathbb{R}^{n}$, Tan $(\eta, x)$ is a $d$-cone. Moreover $\left\{\nu \in \operatorname{Tan}(\eta, x): F_{1}(\nu)=1\right\}$ is closed under weak convergence (see [19] $2.3)$.

Definition 2.6. The basis of a $d$-cone $\mathcal{M}$ of Radon measures is the set $\{\Psi \in \mathcal{M}$ : $\left.F_{1}(\Psi)=1\right\}$. We say that $\mathcal{M}$ has a closed (respectively compact) basis if its basis is closed (respectively compact) in the topology induced by the metric

$$
\sum_{p=0}^{\infty} 2^{-p} \min \left\{1, F_{p}(\Phi, \Psi)\right\}
$$

defined for Radon measures $\Psi$ and $\Phi$.

Proposition 2.1 ([19], Proposition 1.12). The set of Radon measures on $\mathbb{R}^{n}$ with the metric above is a complete separable metric space.

Remark 2.4.

i) As indicated in 1.9(4), Proposition 1.12 and Proposition 1.11 of [19] the notion of convergence in this metric coincides with the notion of weak convergence of Radon measures.

ii) A $d$-cone of Radon measures in $\mathbb{R}^{n}$ has a closed basis if and only if it is a relatively closed subset of the set of non-zero Radon measures in $\mathbb{R}^{n}$.

Proposition 2.2 ([19, Proposition 2.2). Let $\mathcal{M}$ be a d-cone of Radon measures. $\mathcal{M}$ has a compact basis if and only if for every $\lambda \geq 1$ there is $\tau>1$ such that $F_{\tau r}(\Psi) \leq \lambda F_{r}(\Psi)$ for every $\Psi \in \mathcal{M}$ and every $r>0$. In this case $0 \in \operatorname{spt} \Phi$ for all $\Psi \in \mathcal{M}$.

The following theorem is in the same vein as Theorem 2.6 in [19].

Theorem 2.1. Let $\mathcal{F}$ and $\mathcal{M}$ be d-cones. Assume that $\mathcal{F} \subset \mathcal{M}$, that $\mathcal{F}$ is relatively closed with respect to the weak convergence of Radon measures and that $\mathcal{M}$ has a compact basis. Furthermore suppose that the following property holds:

$$
\left\{\begin{array}{l}
\exists \epsilon_{0}>0 \text { such that } \forall \epsilon \in\left(0, \epsilon_{0}\right) \text { there exists no } \mu \in \mathcal{M} \text { satisfying } \\
d_{r}(\mu, \mathcal{F}) \leq \epsilon \quad \forall r \geq r_{0}>0 \text { and } d_{r_{0}}(\mu, \mathcal{F})=\epsilon .
\end{array}\right.
$$

Then for a Radon measure $\eta$ and $x \in \operatorname{spt} \eta$ if

$$
\operatorname{Tan}(\eta, x) \subset \mathcal{M} \text { and } \operatorname{Tan}(\eta, x) \cap \mathcal{F} \neq \emptyset, \quad \text { then } \operatorname{Tan}(\eta, x) \subset \mathcal{F} .
$$

Corollary 2.1. Let $\mathcal{F}$ and $\mathcal{M}$ be d-cones. Assume that $\mathcal{F} \subset \mathcal{M}$, that $\mathcal{F}$ is relatively closed with respect to the weak convergence of Radon measures and that $\mathcal{M}$ has a compact basis. Furthermore suppose that there exists $\epsilon_{0}>0$ such that if $d_{r}(\mu, \mathcal{F})<$ $\epsilon_{0}$ for all $r \geq r_{0}>0$, then $\mu \in \mathcal{F}$. Then for a Radon measure $\eta$ and $x \in \operatorname{spt} \eta$ if

$$
\operatorname{Tan}(\eta, x) \subset \mathcal{M} \text { and } \operatorname{Tan}(\eta, x) \cap \mathcal{F} \neq \emptyset, \quad \text { then } \operatorname{Tan}(\eta, x) \subset \mathcal{F} .
$$


Note that the condition stated in Corollary 2.1] is stronger than condition (P), and a simple argument shows it.

Proof of Theorem 2.1. We proceed by contradiction; i.e. assume that $\operatorname{Tan}(\eta, x) \subset$ $\mathcal{M}, \operatorname{Tan}(\eta, x) \cap \mathcal{F} \neq \emptyset$ but there exists $\nu \in \operatorname{Tan}(\eta, x) \backslash \mathcal{F}$. Since $\mathcal{F}$ is closed there exists $\epsilon_{1} \in\left(0, \frac{1}{2} \min \left\{\epsilon_{0}, 1\right\}\right)$ such that $d_{1}(\eta, \mathcal{F})>2 \epsilon_{1}$. Moreover there exist $s_{i} \searrow 0$ and $c_{i}>0$ such that $c_{i} T_{x, s_{i}}[\eta] \rightarrow \nu$. Since $\operatorname{Tan}(\eta, x) \cap \mathcal{F} \neq \emptyset$ there also exist $\delta_{i}>0$ and $r_{i} \searrow 0$ such that $\delta_{i} T_{x, r_{i}}[\eta] \rightarrow \widetilde{\nu} \in \mathcal{F}$. Thus for $i$ large enough

$$
d_{1}\left(T_{x, r_{i}}[\eta], \mathcal{F}\right)=d_{1}\left(\delta_{i} T_{x, r_{i}}[\eta], \mathcal{F}\right)<\epsilon_{1} \text { and } d_{1}\left(T_{x, s_{i}}[\eta], \mathcal{F}\right)>\epsilon_{1} .
$$

Without loss of generality we may assume that $s_{i}<r_{i}$. Let $\tau_{i} \in\left(\frac{s_{i}}{r_{i}}, 1\right)$ be the largest number such that $\tau_{i} r_{i}=\rho_{i}$ satisfies

$$
d_{1}\left(T_{x, \rho_{i}}[\eta], \mathcal{F}\right)=\epsilon_{1} .
$$

Hence for all $\alpha \in\left(\tau_{i}, 1\right)$

$$
d_{1}\left(T_{x, \alpha r_{i}}[\eta], \mathcal{F}\right)=d_{\alpha / \tau_{i}}\left(T_{x, \rho_{i}}[\eta], \mathcal{F}\right)<\epsilon_{1} .
$$

We claim that $\tau_{i} \rightarrow 0$ as $i \rightarrow \infty$. In fact, otherwise there exists a subsequence $\tau_{i_{k}} \rightarrow \tau \in(0,1)$, and $\delta_{i_{k}} T_{x, \rho_{i_{k}}}[\eta]=\delta_{i_{k}} T_{x, \tau_{i_{k}} r_{i_{k}}}[\eta] \rightarrow T_{0, \tau}[\widetilde{\nu}] \in \mathcal{F}$, which implies that $d_{1}\left(T_{x, \rho_{i_{k}}}[\eta], \mathcal{F}\right) \rightarrow 0$ as $i_{k} \rightarrow \infty$, which contradicts (2.16). Therefore (2.16) and (2.17) yield

$$
\lim _{i \rightarrow \infty} d_{1}\left(T_{x, \rho_{i}}[\eta], \mathcal{F}\right)=\epsilon_{1}
$$

and for every $r>1$,

$$
\limsup _{i \rightarrow \infty} d_{r}\left(T_{x, \rho_{i}}[\eta], \mathcal{F}\right) \leq \epsilon_{1}
$$

Note that $F_{r}\left(T_{x, \rho_{i}}[\eta]\right)=\frac{1}{\rho_{i}} F_{B\left(x, r \rho_{i}\right)}(\eta) \in(0, \infty)$ for $x \in$ spt $\eta$. Moreover a simple calculation shows that for $i$ large enough

$$
0<\frac{r}{2} \eta\left(B\left(x, \frac{r \rho_{i}}{2}\right)\right) \leq F_{r}\left(T_{x, \rho_{i}}[\eta]\right) \leq r \eta\left(B\left(x, r \rho_{i}\right)\right) \leq r \eta(B(x, r))<\infty .
$$

Since $\epsilon_{1}<1, \lambda=\frac{2}{1+\epsilon_{1}}>1$, and by Proposition 2.2 there is $\tau>1$ so that $F_{\tau r}(\Psi) \leq \lambda F_{r}(\Psi)$ for every $\Psi \in \mathcal{M}$ and every $r>0$. For $r \geq 1$ and $i$ large enough there is $\Psi \in \mathcal{M}$ so that $F_{\tau r}(\Psi)=1$ and

$$
F_{r}\left(\frac{T_{x, \rho_{i}}[\eta]}{F_{r \tau}\left(T_{x, \rho_{i}}[\eta]\right)}, \Psi\right) \leq F_{\tau r}\left(\frac{T_{x, \rho_{i}}[\eta]}{F_{r \tau}\left(T_{x, \rho_{i}}[\eta]\right)}, \Psi\right) \leq \epsilon_{1} .
$$

Hence

$$
\frac{F_{r}\left(T_{x, \rho_{i}}[\eta]\right)}{F_{\tau r}\left(T_{x, \rho_{i}}[\eta]\right)} \geq F_{r}(\Psi)-\epsilon_{1} \geq \frac{1+\epsilon_{1}}{2} F_{\tau r}(\Psi)-\epsilon_{1}=\frac{1-\epsilon_{1}}{2} .
$$

Thus for $p=1,2, \ldots(2.22)$ yields

$$
\frac{F_{\tau^{p}}\left(T_{x, \rho_{i}}[\eta]\right)}{F_{1}\left(T_{x, \rho_{i}}[\eta]\right)} \leq\left(\frac{1-\epsilon_{1}}{2}\right)^{-p} .
$$

Combining (2.20), (2.23) and i) in Remark 2.1 we conclude that for $p=1,2, \ldots$, $\tau>1$ (as above) and $i$ large enough,

$$
\frac{T_{x, \rho_{i}}[\eta]\left(B\left(0, \tau^{p} / 2\right)\right)}{F_{1}\left(T_{x, \rho_{i}}[\eta]\right)} \leq 2\left(\frac{2}{1-\epsilon_{1}}\right)^{p} \tau^{-p} \leq 2\left(\frac{2}{\left(1-\epsilon_{1}\right) \tau}\right)^{p} .
$$


Thus for any $s>0,(2.24)$ ensures that

$$
\limsup _{i \rightarrow \infty} \frac{T_{x, \rho_{i}}[\eta](B(0, s))}{F_{1}\left(T_{x, \rho_{i}}[\eta]\right)}<\infty .
$$

By the compactness theorem for Radon measures there exists a subsequence $i_{k}$ such that $\frac{T_{x, \rho_{k_{k}}}[\eta]}{F_{1}\left(T_{x, \rho_{i}}[\eta]\right)}$ converges to a Radon measure $\Phi \in \mathcal{M}$ (as $\mathcal{M}$ has a closed basis), satisfying $F_{1}(\Phi)=1$. Therefore $F_{r}(\Phi)>0$ for $r \geq 1$.

Combining iii) in Remark 2.2 with (2.18) and (2.19) we have that

$$
d_{1}(\Phi, \mathcal{F})=\epsilon_{1}
$$

$$
d_{r}(\Phi, \mathcal{F}) \leq \epsilon_{1} \text { for all } r>1 .
$$

Since $\epsilon_{1}<\epsilon_{0} / 2$, (2.26) and (2.27) contradict condition (P). This concludes the proof of Theorem 2.1.

We next recall a couple of results from [19] and [18. They provide additional information about $\operatorname{Tan}(\Phi, x)$ for a Radon measure $\Phi$ and for $x \in \operatorname{spt} \Phi$. The first result yields conditions that ensure that $\operatorname{Tan}(\Phi, x)$ has a compact basis. As we will see these conditions are satisfied by the harmonic measures considered in this paper. The second result states that tangent measures to tangent measures of $\Phi$ are tangent measures of $\Phi$.

Theorem 2.2 (19, Corollary 2.7). Let $\Phi$ be a Radon measure in $\mathbb{R}^{n}$, and let $x \in \operatorname{spt} \Phi$. Then Tan $(\Phi, x)$ has a compact basis if and only if

$$
\limsup _{r \rightarrow 0} \frac{\Phi(B(x, 2 r))}{\Phi(B(x, r))}<\infty .
$$

Theorem 2.3 (18], Theorem 14.16). Let $\Phi$ be a Radon measure in $\mathbb{R}^{n}$. Then $\Phi$ a.e. $a \in \mathbb{R}^{n}$, if $\Psi \in \operatorname{Tan}(\Phi, a)$,

i) $T_{x, \rho}[\Psi] \in \operatorname{Tan}(\Phi, a)$ for all $x \in \operatorname{spt} \Psi$ and all $\rho>0$.

ii) $\operatorname{Tan}(\Psi, x) \subset \operatorname{Tan}(\Phi, a)$ for all $x \in \operatorname{spt} \Psi$.

Finally we present a couple of results which will be used later in the paper.

Definition 2.7. A Radon measure $\omega$ in $\mathbb{R}^{n}$ is said to be locally doubling if for every compact set $K \subset$ spt $\omega$ there exists $C=C_{k} \geq 1$ and $R_{K}=R>0$ such that for $Q \in K$ and $s \in(0, R)$,

$$
\omega(B(Q, 2 s)) \leq C \omega(B(Q, s)) .
$$

Lemma 2.3. Let $\omega$ be a locally doubling measure in $\mathbb{R}^{n}$. Let $\Psi$ be a non-zero Radon measure with $\Psi \in \operatorname{Tan}(\omega, Q)$. There exists a sequence of positive numbers $\left\{r_{i}\right\}_{i \geq 1}$ with $\lim _{i \rightarrow \infty} r_{i}=0$ such that $r_{i}{ }^{-1}(\operatorname{spt} \omega-Q)$ converges to spt $\Psi$ in the Hausdorff distance sense uniformly on compact sets.

Proof. Since $\Psi \in \operatorname{Tan}(\omega, Q)$ and $\omega$ is locally doubling by Remark (3) in 14.4 of [18, we have that there are a sequence $r_{i} \downarrow 0$ and a positive constant $c$ such that $\Psi=c \lim _{i \rightarrow \infty} \omega\left(B\left(Q, r_{i}\right)\right)^{-1} T_{Q, r_{i}}[\omega]$. Let $X=\lim _{i \rightarrow \infty} X_{i} \in B\left(0, R_{0}\right)$ where $X_{i}=$ $r_{i}^{-1}\left(Z_{i}-Q\right)$ with $Z_{i} \in \operatorname{spt} \omega$. For $r \in(0,1)$ there exists $i_{0} \geq 1$ such that for $i \geq i_{0}$, $\left|X-X_{i}\right|<\frac{r}{2}$ and $\left|Z_{i}-Q\right| \leq r_{i}\left|X_{i}\right| \leq r_{i}(|X|+1) \leq R_{0}+1$. Since $\omega$ is locally doubling there exists $C_{0} \geq 1$ and $R>0$ such that for $P \in B\left(Q, 2\left(R_{0}+1\right)\right)$ and 
$s<R, \omega(B(P, 2 s)) \leq C_{0} \omega(B(P, s))$. Thus for $r \leq \min \{R, 1\}$ and $i$ large enough so that $r_{i}\left(R_{0}+1\right)<R$, we have

$$
\begin{aligned}
\frac{T_{Q, r_{i}}[\omega](B(x, r))}{\omega\left(B\left(Q, r_{i}\right)\right)} & =\frac{\omega\left(B\left(Q+r_{i} X, r r_{i}\right)\right)}{\omega\left(B\left(Q, r_{i}\right)\right)} \\
& \geq \frac{\omega\left(B\left(Q+r_{i} X_{i}, r_{i}\left(r-\left|X-X_{i}\right|\right)\right)\right.}{\omega\left(B\left(Q, r_{i}\right)\right)} \\
& \geq \frac{\omega\left(B\left(Z_{i}, \frac{r r_{i}}{2}\right)\right)}{\omega\left(B\left(Z_{i}, r_{i}\left(R_{0}+2\right)\right)\right)} \geq C_{0}^{k}>0,
\end{aligned}
$$

where $k \in \mathbb{N}$ is such that $2^{-k}\left(R_{0}+2\right) \leq \frac{r}{2}<2^{-(k-1)}\left(R_{0}+2\right)$. Thus

$$
\begin{aligned}
\Psi(B(x, 2 r)) & \geq \Psi(\overline{B(x, r)}) \\
& \geq \limsup _{i \rightarrow \infty} c \frac{T_{a, r_{i}}[\omega](\overline{B(x, r))}}{\omega\left(B\left(Q, r_{i}\right)\right)} \geq C_{0}^{k}>0 .
\end{aligned}
$$

Thus (2.31) ensures that $x \in \operatorname{spt} \Psi$. This shows that $\lim _{i \rightarrow \infty} r_{i}^{-1}(\operatorname{spt} \omega-Q) \subset$ spt $\Psi$. To show the opposite inclusion assume that for $X \notin \lim _{i \rightarrow \infty} r_{i}^{-1}(\operatorname{spt} \omega-Q$ ) there exists $\left\{r_{i_{k}}\right\} \subset\left\{r_{i}\right\}, r_{i_{k}} \searrow 0$ such that $d\left(X, r_{i_{k}}^{-1}\right.$ (spt $\left.\left.\omega-Q\right)\right) \geq \epsilon_{0}$. Thus $B\left(x, \frac{\epsilon_{0}}{2}\right) \cap r_{i_{k}}^{-1}(\operatorname{spt} \omega-Q)=\emptyset$. For $\varphi \in C_{c}^{\infty}\left(B\left(X, \frac{\epsilon_{0}}{2}\right)\right)$ we have

$$
\int \varphi d \Psi=C \lim _{i_{k} \rightarrow \infty} \frac{1}{\omega\left(B\left(Q, r_{i_{k}}\right)\right)} \int \varphi\left(\frac{Y-Q}{r_{i_{k}}}\right) d \omega=0,
$$

which ensures that $X \notin \operatorname{spt} \Psi$.

The following lemma is a simple geometric measure theory fact which allows us to give an estimate on the Hausdorff dimension of sets which approach $(n-1)$-planes locally.

Lemma 2.4. Let $\Sigma \subset \mathbb{R}^{n}$ be such that $\forall Q \in \Sigma$

$$
\lim _{r \rightarrow 0} \beta_{\Sigma}(Q, r)=0, \text { where } \beta_{\Sigma}(Q, r)=\inf _{L \in G(n, n-1)} \sup _{y \in B(Q, r) \cap \Sigma} \frac{d(y, L)}{r} .
$$

Then

$$
\operatorname{dim}_{\mathcal{H}} \Sigma \leq n-1
$$

The following proof is an adaptation of the argument used in 20] to prove Lemma 3 in Chapter 3, $\S 4$ of $[20$.

Proof. Let $Q \in \Sigma$. Given $\epsilon>0$, there exists $r_{Q, \epsilon}>0$ such that for $r<r_{Q, \epsilon}$ there exists an $(n-1)$-plane $L(Q, r)$ through $Q$ so that

$$
\Sigma \cap B(Q, r) \subset(L(Q, r) \cap B(Q, r) ; \epsilon r) .
$$

Note that for $\epsilon>0$

$$
\Sigma=\bigcup_{j=1}^{\infty} \Sigma_{j}, \text { where } \Sigma_{j}=\left\{Q \in \Sigma: r_{Q, \epsilon}>\frac{10}{2^{j}}\right\} .
$$

Without loss of generality we may assume that $0 \in \Sigma$. Let $k \in \mathbb{N}$. For $j_{0} \geq 1$ cover $\Sigma_{j_{0}} \cap B(0, k)$ by sets $\left\{C_{s}\right\}_{s \geq 1}$ of diameter less than $\delta>0$. Choosing $\delta<\frac{1}{2^{j_{0}}}$ we can ensure that each such set is contained in a ball of center $Q \in \Sigma_{j_{0}}$ and radius $r_{Q}=\operatorname{diam} C_{s}$ for some $s$ with $Q \in C_{s}$ less than $\delta$, i.e. $r_{Q}<\frac{1}{10} r_{Q, \epsilon}$. Note that 
$B\left(Q, r_{Q}\right) \cap L\left(Q, r_{Q}\right)$ can be covered by $N \epsilon^{-n+1}$ balls $\left\{B_{l}\right\}_{l}$ centered in $L\left(Q, r_{Q}\right)$ with radius $5 \epsilon r_{Q}$ and such that the balls of the same center and radius $\epsilon r_{Q}$ are disjoint. Here $N>0$ is an absolute constant that only depends on $n$. Thus for $\gamma>0$

$$
\sum_{l}\left(\operatorname{diam} B_{l}\right)^{n-1+\gamma}=\left(5 \epsilon r_{Q}\right)^{n-1+\gamma} N \epsilon^{-n+1}=5^{n-1} \epsilon^{\gamma} N r_{Q}^{n-1+\gamma} .
$$

Note that if $\gamma \geq-\frac{\ln \left(4 N 5^{n-1}\right)}{\ln (5 \epsilon)}$, then

$$
\sum_{l}\left(\operatorname{diam} B_{o}\right)^{n-1+\gamma} \leq \frac{1}{4} r_{Q}^{n-1+\gamma} .
$$

Thus for $\delta<\frac{1}{2^{j_{0}}}$

$$
\mathcal{H}_{5 \epsilon \delta}^{n-1+\gamma}\left(\Sigma_{j_{0}} \cap B(0, k)\right) \leq \frac{1}{4} \mathcal{H}_{\delta}^{n-1+\gamma}\left(\Sigma_{j_{0}} \cap B(0, k)\right) .
$$

Letting $\delta \rightarrow 0$ we conclude that $\forall j \in \mathbb{N}$ and $\gamma \geq-\ln \left(4 N 5^{n-1}\right) / \ln (5 \epsilon)$,

$$
\mathcal{H}^{n-1+\gamma}\left(\Sigma_{j} \cap B(0, k)\right)=0 .
$$

Thus (2.36) ensures that

$$
\mathcal{H}^{n-1+\gamma}(\Sigma \cap B(0, k))=0
$$

for $\gamma \geq-\frac{\ln \left(4 N 5^{n-1}\right)}{\ln (5 \epsilon)}$ and $\epsilon>0$. Letting $\epsilon \rightarrow 0$ we conclude that $\mathcal{H}^{n-1+\gamma}(\Sigma \cap B(0, k))=0$ for all $\gamma>0$ and hence $\mathcal{H}^{n-1+\gamma}(\Sigma)=0$ also. This implies that $\operatorname{dim}_{\mathcal{H}} \Sigma \leq n-1$.

\section{TWO-SIDED LOCALLY NON-TANGENTIALLY ACCESSIBLE DOMAINS}

Definition 3.1. A domain $\Omega \subset \mathbb{R}^{n}$ is admissible if

- $\Omega^{+}=\Omega$ and $\omega^{-}=\operatorname{int} \omega^{c}$ are regular for the Dirichlet problem.

- $\partial \Omega^{+}=\partial \Omega^{-}=\partial \Omega$.

- There exist points $X^{ \pm} \in \Omega^{ \pm}$such that for every point $Q \in \partial \Omega$ there exists $0<R<\min \left\{\delta\left(X^{+}\right), \delta\left(X^{-}\right)\right\}$satisfying $u \in C^{0}(B(Q, R)) \cap W^{1,2}(B(Q, R))$, where $\delta(X)=\operatorname{dist}(X, \partial \Omega)$,

$$
\begin{aligned}
& u(X)=G_{+}\left(X, X^{+}\right) \text {for } X \in \Omega^{+}, \\
& u(X)=-G_{-}\left(X, X^{-}\right) \text {for } X \in \Omega^{-}
\end{aligned}
$$

and $G_{ \pm}\left(-, X^{ \pm}\right)$denotes the Green function of $\Omega^{ \pm}$with pole at $X^{ \pm}$.

Notation. If $\Omega$ is admissible so is int $\Omega^{c}$. Let $\Omega$ be an admissible domain; we denote by $\omega^{ \pm}$the harmonic measure of $\Omega^{ \pm}$with pole $X^{ \pm}$. Note that in this case $u^{ \pm}=G_{ \pm}\left(-, X^{ \pm}\right)$.

The monotonicity formula of Alt, Caffarelli and Freidman plays a role in this work. We recall several of the results which will be used later. 
Theorem 3.1 ([1]). Let $\Omega$ be an admissible domain. Then for $Q \in \partial \Omega$ there exists $0<R<\min \left\{\delta\left(X^{+}\right), \delta\left(X^{-}\right)\right\}$such that the quantity

$$
\gamma(Q, r)=\left(\frac{1}{r^{2}} \int_{B(Q, r)} \frac{\left|\nabla u^{+}\right|^{2}}{|X-Q|^{n-2}} d X\right) \cdot\left(\frac{1}{r^{2}} \int_{B(Q, r)} \frac{\left|\nabla u^{-}\right|^{2}}{|X-Q|^{n-2}} d X\right)
$$

is an increasing function of $r$ for $r \in(0, R)$ and $\gamma(Q, R)<\infty$.

Note that the ACF-monotonicity formula ensures that

$$
\gamma(Q)=\lim _{r \rightarrow 0} \gamma(Q, r)
$$

exists and is a non-negative finite quantity. A combination of the results of AltCaffarelli-Friedman, Beckner-Kenig-Pipher and Brothers-Ziemer asserts that if $\gamma(Q)>0$, then all blow-ups of the boundary at $Q$ are $(n-1)$-planes (see [1, [2] and [5]). This last fact will not be used here.

Our immediate goal is to estimate $\gamma(Q, r)$ in terms of $\omega^{ \pm}$and $u^{ \pm}$. Let $\varphi \in$ $C_{c}^{\infty}\left(\mathbb{R}^{n}\right)$. The harmonic extension $v_{\varphi}$ of $\varphi$ to $\Omega$ (i.e. $\Delta v_{\varphi}=0$ in $\Omega$ and $v_{\varphi}=\varphi$ in $\partial \Omega)$ satisfies for $u \notin \operatorname{spt} \varphi$

$$
v_{\varphi}(Y)=-\int\langle\nabla G(Y, X), \nabla \varphi(X)\rangle d X .
$$

Let $R<\min \left\{\delta\left(X^{+}\right), \delta\left(X^{-}\right)\right\}, 2 r<R, Q \in \partial \Omega$ and $\varphi=1$ on $B\left(Q, \frac{3 r}{2}\right), \varphi=0$ on $B(Q, 2 r)^{c}, 0 \leq \varphi \leq 1$ and $|\nabla \varphi| \leq \frac{C}{r}$. By the maximum principle $v_{\varphi}^{ \pm}\left(X^{ \pm}\right) \geq$ $\omega^{ \pm}(B(Q, r))$. Here $v_{\varphi}^{ \pm}$denotes the harmonic extension of $\varphi$ to $\Omega^{ \pm}$. Hence by (3.4) we have

$$
\begin{aligned}
\omega^{ \pm}(B(Q, r)) \leq & \frac{C}{r} \int_{B(Q, 2 r) \backslash B(Q, r)}\left|\nabla u^{ \pm}\right| \\
\leq & \frac{C}{r}\left(\int_{B(Q, 2 r) \backslash B(Q, r)} \frac{\left|\nabla u^{ \pm}\right|^{2}}{|X-Q|^{n-2}}\right)^{1 / 2} \\
& \cdot\left(\int_{B(Q, 2 r) \backslash B(Q, r)}|X-Q|^{n-2}\right)^{1 / 2},
\end{aligned}
$$

which yields

$$
\frac{\omega^{ \pm}(B(Q, r))}{r^{n-1}} \leq C\left(\frac{1}{r^{2}} \int_{B(Q, 2 r) \backslash B(Q, r)} \frac{\left|\nabla u^{ \pm}\right|^{2}}{|X-Q|^{n-2}}\right)^{1 / 2}
$$

and

$$
\frac{\omega^{+}(B(Q, r))}{r^{n-1}} \cdot \frac{\omega^{-}(B(Q, r))}{r^{n-1}} \leq C \gamma(Q, 2 r)^{1 / 2}
$$

Note that $\Delta\left(u^{ \pm}\right)^{2}=2\left|\nabla u^{ \pm}\right|^{2} \geq 0$ because $u^{ \pm}$is zero on the support of the measure $\Delta u^{ \pm}$. Using Cacciopoli's inequality as well as the fact that $\left(u^{ \pm}\right)^{2}$ is subharmonic (and therefore the averages over spheres are increasing as a function of 
the radius) we have for $Q \in \partial \Omega$ that

$$
\begin{aligned}
& \int_{B(Q, r)} \frac{\left|\nabla u^{ \pm}\right|^{2}}{|X-Q|^{n-2}}=\frac{1}{2} \int_{B(Q, r)} \frac{\Delta\left(u^{ \pm}\right)^{2}}{|X-Q|^{n-2}} \\
& \quad=C_{n}\left(u^{ \pm}\right)^{2}(Q)+\frac{1}{r^{n-2}} \int_{\partial B(Q, r)} u^{ \pm} \frac{\partial u^{ \pm}}{\partial r}+\frac{n-2}{2 r^{n-1}} \int_{\partial B(Q, r)}\left(u^{ \pm}\right)^{2} \\
& \quad=\frac{1}{r^{n-2}} \int_{B(Q, r)}\left|\nabla u^{ \pm}\right|^{2}+\frac{n-2}{2 r^{n-1}} \int_{\partial B(Q, r)}\left(u^{ \pm}\right)^{2} \\
& \quad \leq C \frac{1}{r^{n}} \int_{B(Q, 2 r)}\left(u^{ \pm}\right)^{2}+\frac{n-2}{2 r^{n-1}} \int_{\partial B(Q, r)}\left(u^{ \pm}\right)^{2} \\
& \quad \leq C \frac{1}{r^{n}} \int_{B(Q, 2 r)}\left(u^{ \pm}\right)^{2}+\frac{n-2}{2 r^{n}} \int_{r}^{2 r}\left(\int_{\partial B(Q, s)}\left(u^{ \pm}\right)^{2}\right) d s \\
& \quad \leq C \frac{1}{r^{n}} \int_{B(Q, 2 r)}\left(u^{ \pm}\right)^{2} .
\end{aligned}
$$

We have proved the following result:

Lemma 3.1. Assume given $\Omega \subset \mathbb{R}^{n}$, an admissible domain. Let $R<\min \left\{\delta\left(X^{+}\right)\right.$, $\left.\delta\left(X^{-}\right)\right\}, 4 r<R$, and $Q \in \partial \Omega ;$ then

$$
\frac{\omega^{ \pm}(B(Q, r))}{r^{n-1}} \leq C\left(\frac{1}{r^{2}} \int_{B(Q, 2 r)} \frac{\left|\nabla u^{ \pm}\right|^{2}}{|X-Q|^{n-2}}\right)^{1 / 2} \leq C\left(\frac{1}{r^{n+2}} \int_{B(Q, 4 r)}\left(u^{ \pm}\right)^{2}\right)^{1 / 2} .
$$

Therefore

$$
\begin{aligned}
\frac{\omega^{+}(B(Q, r))}{r^{n-1}} \cdot \frac{\omega^{-}(B(Q, r))}{r^{n-1}} \leq & C \gamma(Q, 2 r)^{1 / 2} \\
\gamma(Q, 2 r) \leq & C\left(\frac{1}{r^{n+2}} \int_{B(Q, 4 r)}\left(u^{+}\right)^{2}\right) \\
& \cdot\left(\frac{1}{r^{n+2}} \int_{B(Q, 4 r)}\left(u^{-}\right)^{2}\right) .
\end{aligned}
$$

Remark 3.1. For $Q_{0} \in \partial \Omega, r_{0}<\frac{R}{8}$ and $Q \in B\left(Q_{0}, \frac{r_{0}}{2}\right) \cap \partial \Omega$, we have for $r<\frac{r_{0}}{2}$,

$$
\begin{aligned}
\gamma(Q, r) & \leq \gamma\left(Q, \frac{r_{0}}{2}\right) \\
& \leq C\left(\frac{1}{r_{0}^{n+2}} \int_{B\left(Q, r_{0}\right)}\left(u^{+}\right)^{2}\right) \cdot\left(\frac{1}{r_{0}^{n+2}} \int_{B\left(Q, r_{0}\right)}\left(u^{-}\right)^{2}\right) .
\end{aligned}
$$

Moreover

$$
\frac{\omega^{+}(B(Q, r))}{r^{n-1}} \cdot \frac{\omega^{-}(B(Q, r))}{r^{n-1}} \leq C \gamma(Q, 2 r)^{\frac{1}{2}} \leq \frac{C}{r_{0}^{n+2}}\|u\|_{L^{2}\left(B\left(Q_{0}, 4 r_{0}\right)\right)}^{2} .
$$

Here $C$ only depends on $n$. Thus Beurling's inequality (see 9] Chapter IV, Theorem 6.2 and Chapter VI, proof of Theorem 6.3) holds in higher dimensions.

Definition 3.2. A domain $\Omega \subset \mathbb{R}^{n}$ satisfies the corkscrew condition if for each compact set $K \subset \mathbb{R}^{n}$ there exists $R>0$ such that for $Q \in \partial \Omega \cap K$ and $r \in(0, R]$ there exists $A=A(Q, r) \in \Omega$ such that $M^{-1} r<|A-Q|<r$ and $d(A, \partial \Omega)>M^{-1} r$. If $\Omega$ is unbounded we require that $R=\infty$. 
Definition 3.3. A domain $\Omega \subset \mathbb{R}^{n}$ is locally non-tangentially accessible (NTA) if

(1) $\Omega^{ \pm}$satisfies the corkscrew condition.

(2) Harnack Chain Condition. Given a compact set $K \subset \mathbb{R}^{n}$, there exists $R=$ $R_{K}>0$ and $M=M_{K}>1$ such that if $\epsilon>0$, and $X_{1}, X_{2} \in \Omega \cap B\left(Q, \frac{r}{4}\right)$ for some $Q \in \partial \Omega \cap K, r<R, d\left(X_{j}, \partial \Omega\right)>\epsilon$ and $\left|X_{1}-X_{2}\right|<2^{k} \epsilon$, then there exists a Harnack chain from $X_{1}$ to $X_{2}$ of length $M k$ and such that the diameter of each ball is bounded below by $M^{-1} \min \left\{\operatorname{dist}\left(X_{1}, \partial \Omega\right)\right.$, dist $\left.\left(X_{2}, \partial \Omega\right)\right\}$. If $\Omega$ is unbounded we require that $R=\infty$.

If $\Omega$ is bounded and locally NTA, then $\Omega$ is NTA as defined in [11].

In particular since most of the results concerning the behaviour of non-negative harmonic measures on NTA domains are local, suitable modifications hold for locally NTA domains. We briefly summarize the most important ones in the current context.

Lemma 3.2 (11, Lemma 4.1). Let $\Omega$ be a locally NTA domain. Given a compact set $K \subset \mathbb{R}^{n}$, there exists $\beta>0$ such that for all $Q \in \partial \Omega \cap K, 0<2 r<R_{K}$, and every positive harmonic function $u$ in $\Omega \cap B(Q, 2 r)$, which vanishes continuously on $B(Q, 2 r)$, then for $X \in \Omega \cap B(Q, r)$

$$
u(X) \leq C\left(\frac{|X-Q|}{r}\right)^{\beta} \sup \{u(Y): Y \in \partial B(Q, 2 r) \cap \Omega\} .
$$

Here $C$ only depends on $K$.

Lemma 3.3 (11, Lemma 4.4). Let $\Omega$ be a locally NTA domain. Given a compact set $K \subset \mathbb{R}^{n}$ and $0<2 r<R_{K}$, if $u \geq 0$ is a harmonic function in $\Omega \cap B(Q, 4 r)$ and $u$ vanishes continuously on $B(Q, 2 r) \cap \partial \Omega$, then, for $Q \in \partial \Omega \cap K$,

$$
u(Y) \leq C u(A(Q, r))
$$

for all $Y \in B(Q, r) \cap \Omega$. Here $C$ only depends on $K$.

Lemma 3.4 (11, Lemma 4.8). Let $\Omega$ be a locally NTA domain. Given a compact set $K \subset \mathbb{R}^{n}$, for $Q \in \partial \Omega \cap K, 0<2 r<R_{K}$ and $X \in \Omega \backslash B(Q, 2 r)$, then

$$
C^{-1}<\frac{\omega^{X}(B(Q, r))}{r^{n-2} G(A(Q, r), X)}<C,
$$

where $G(A(Q, r), X)$ is the Green function of $\Omega$ with pole $X$.

Lemma 3.5 (11, Lemmas 4.8, 4.11). Let $\Omega$ be a locally NTA domain. Given a compact set $K \subset \mathbb{R}^{n}$, if $M>1$ and $R>0$ are as in Definition 3.3 for $Q \in \partial \Omega \cap K$, $0<2 r<R$, and $X \in \Omega \backslash B(Q, 2 M r)$, then for $s \in[0, r]$

$$
\omega^{X}(B(Q, 2 s)) \leq C \omega^{X}(B(Q, s)),
$$

where $C \geq 1$ only depends on $K$.

Definition 3.4. A domain $\Omega \subset \mathbb{R}^{n}$ is 2 -sided locally non-tangentially accessible if $\Omega^{ \pm}$are both locally NTA.

Lemma 3.6. Let $\Omega \subset \mathbb{R}^{n}$ be a 2-sided locally NTA domain; then $\Omega$ is an admissible domain. 
Proof. Lemmas 3.2 and 3.3 ensure that there exists $M>1$ depending on the NTA constants of $\Omega^{ \pm}$such that for $X^{ \pm} \in \Omega^{ \pm}$and for $r<\frac{1}{M} \min \left\{\delta\left(X^{+}\right), \delta\left(X^{-}\right)\right\}$, if $X \in \Omega^{ \pm} \cap B(Q, r)$, then

$$
G_{ \pm}\left(X, X^{ \pm}\right) \leq C G_{ \pm}\left(A^{ \pm}(Q, r), X^{ \pm}\right)\left(\frac{\delta(X)}{R}\right)^{\beta}
$$

where $\beta$ and $C$ depend on $n$ and the NTA constants of $\Omega^{ \pm}$.

Thus $u=G_{+}\left(-, X^{+}\right)-G_{-}\left(-, X^{-}\right) \in C^{0}(B(Q, R))$. Recall that for $X \in$ $B(Q, r) \cap \Omega^{ \pm}$

$$
\left|\nabla G_{ \pm}\left(X, X^{ \pm}\right)\right| \leq C_{n} \frac{G_{ \pm}\left(X, X^{ \pm}\right)}{\delta(X)} .
$$

We claim that there exist $\eta>0$ and $R \in\left(0, R_{0}\right)$ so that

$$
\int_{B(Q, r)}\left(\frac{G_{ \pm}\left(X, X^{ \pm}\right)}{\delta(X)}\right)^{2+\eta} d X<\infty \quad \text { for } r<R
$$

Note that

$$
\begin{aligned}
& \int_{B(Q, r)}\left(\frac{G_{ \pm}\left(X, X^{ \pm}\right)}{\delta(X)}\right)^{2+\eta} d X \\
&=\sum_{j=0}^{\infty} \int_{\left\{2^{-s-1} r \leq \delta(X)<2^{-j} r\right\}}\left(\frac{G_{ \pm}\left(X, X^{ \pm}\right)}{\delta(X)}\right)^{2+\eta}
\end{aligned}
$$

covers

$$
\left\{X \in B(Q, r) ; 2^{-j-1} r \leq \delta(X)<2^{-j} r\right\} \cap \Omega^{ \pm}=A_{j}^{ \pm}
$$

by balls $\left\{B^{ \pm}\left(X_{i}^{j}, \frac{r}{2^{j-2}}\right)\right\}_{i=1}^{N_{j}}$ such that $X_{i}^{j} \in A_{j}^{ \pm},\left|X_{i}^{j}-X_{l}^{j}\right| \geq \frac{r}{2^{j-2}}$ for $i \neq l$. These balls have finite overlaps bounded by a number which only depends on $n$. Moreover

$$
\operatorname{dist}\left(B^{ \pm}\left(X_{i}^{j}, \frac{r}{2^{j-2}}\right) \cap A_{j}^{+}, \partial \Omega\right) \geq \frac{r}{2^{j+2}} .
$$

Note that for $X \in A_{j}^{+}(3.13)$ yields

$$
G_{+}\left(X, X^{+}\right) \leq C G_{+}\left(A^{+}(Q, r) ; X^{+}\right) 2^{-j \beta}
$$

and

$$
\begin{gathered}
\int_{2^{-j-1} r \leq \delta(X)<2^{-j} r}\left(\frac{G\left(X, X^{+}\right)}{\delta(X)}\right)^{2+\eta} d X \\
\leq C r^{-(1+\eta)} 2^{j(1+\eta)} 2^{-j \beta(1+\eta)} G_{+}\left(A^{+}(Q, r), X^{+}\right)^{1+\eta} \\
\cdot \int_{2^{-j-1} r \leq \delta(X)<2^{-j} r} \frac{G\left(X, X^{+}\right)}{\delta(X)} d X .
\end{gathered}
$$

For $X \in B^{+}\left(X_{i}^{j}, \frac{r}{2^{j+2}}\right)$ [11, Lemma 4.8] yields

$$
\frac{G_{+}\left(X, X^{+}\right)}{\delta(X)} \sim \frac{\omega^{+}\left(B\left(Q_{X}, \delta(X)\right)\right.}{\delta(X)^{n-1}},
$$

where $Q_{X} \in \partial \Omega$ is such that $\left|X-Q_{X}\right|=\delta(X)$. The notation $a \sim b$ means that there exists a constant $C>1$ such that $C^{-1} \leq a / b \leq c$. By Harnack's principle for 
$X \in B^{+}\left(X_{i}^{j}, \frac{r}{2^{j-2}}\right) \cap A_{j}^{+}$,

$$
G_{+}\left(X, X^{+}\right) \sim G_{+}\left(X_{i}^{j}, X^{+}\right) .
$$

Note also that for $X \in B^{+}\left(X_{i}^{j}, \frac{r}{2^{j-2}}\right) \cap A_{j}^{+}, \delta(X) \sim \frac{r}{2^{j}} \sim \delta\left(X_{i}^{j}\right)$. Combining this remark with the doubling property of $\omega^{ \pm}$(see [11], $4.9 \& 4.11$ ), (3.23) and (3.24) we obtain that for $X \in B^{+}\left(X_{i}^{j}, \frac{r}{2^{j-2}}\right) \cap A_{j}^{+}$

$$
\frac{G_{+}\left(X, X^{+}\right)}{\delta(X)} \sim \frac{G_{+}\left(X_{i}^{j}, X^{+}\right)}{\delta\left(X_{i}^{j}\right)} \sim \frac{\omega^{+}\left(B\left(Q_{i}^{j}, \frac{r}{2^{j}}\right)\right)}{\left(\frac{r}{2^{i}}\right)^{n-1}}
$$

where $Q_{i}^{j} \in \partial \Omega$ is such that $\delta\left(X_{i}^{j}\right)=\left|X_{i}^{j}-Q_{i}^{j}\right|$. In particular

$$
\begin{aligned}
\left|Q_{i}^{j}-Q_{l}^{j}\right| & \geq\left|X_{i}^{j}-X_{l}^{j}\right|-\left|X_{i}^{j}-Q_{i}^{j}\right|-\left|X_{l}^{j}-Q_{l}^{j}\right| \\
& \geq\left|X_{i}^{j}-X_{l}^{j}\right|-\frac{r}{2^{j}} \geq \frac{r}{2^{j-2}}-\frac{r}{2^{j}} \geq \frac{r}{2^{j}} .
\end{aligned}
$$

Thus $\left\{B\left(Q_{i}^{j} ; \frac{r}{2^{j}}\right)\right\}_{i=1}^{N_{j}}$ is a disjoint family of balls in $B(Q, 2 r)$. Hence the doubling property of $\omega^{+}$and (3.25) yield

$$
\begin{aligned}
& \int_{2^{-j-1}} \leq \delta(X)<2^{-j} r \frac{G_{+}\left(X, X^{+}\right)}{\delta(X)} d X \\
&= \sum_{j=1}^{N_{j}} \int_{A_{j}^{+} \cap B^{+}\left(X_{i}^{j}, \frac{r}{2^{j-2}}\right)} \frac{G_{+}\left(X, X^{+}\right)}{\delta(X)} d X \\
& \leq C \sum_{i=1}^{N_{j}} \frac{\omega^{+}\left(B\left(Q_{i}^{j}, r 2^{-j}\right)\right)}{\left(r 2^{-j}\right)^{n-1}} \mathcal{H}^{n}\left(A_{j}^{+} \cap B^{+}\left(X_{i}^{j}\right)\right) \\
& \leq C \frac{\left(r 2^{-j}\right)^{n}}{\left(r 2^{-j}\right)^{n-1}} \omega^{+}(B(Q, r)) \\
& \leq C 2^{-j} r \omega^{+}(B(Q, r)) .
\end{aligned}
$$

Combining (3.20), (3.22) and (3.26) we obtain

$$
\begin{gathered}
\int_{B(Q, r)}\left(\frac{G_{+}\left(X, X^{+}\right)}{\delta(X)}\right)^{2+\eta} d X \\
\leq C \sum_{j=0}^{\infty} 2^{j(1+\eta)-j \beta(1+\eta)} 2^{-j} \cdot\left(\frac{G_{+}\left(A^{+}(Q, r), X^{+}\right)}{r}\right)^{1+\eta} \cdot r \omega^{+}(B(Q, r)) .
\end{gathered}
$$

If $\eta<\frac{\beta}{1-\beta}$, the series on the r.h.s. in (3.27) converges. The estimate for $G_{-}\left(-, X^{-}\right)$ is identical. Thus using (3.18), (3.15) and (3.27) we conclude that

$$
\int_{B(Q, r)}|\nabla u|^{2+\eta} \leq C_{\eta}\left(\frac{\omega^{+}(B(Q, r))^{2+\eta}}{r^{(n-1)(1+\eta)-1}}+\frac{\omega^{-}(B(Q, r))^{2+\eta}}{r^{(n-1)(1+\eta)}}\right) .
$$

Hence $u \in W^{1,2}(B(Q, r))$ and $\Omega$ is admissible. 
Theorem 3.2. Let $\Omega \subset \mathbb{R}^{n}$ be a 2-sided locally NTA domain. Given a compact set $K \subset \mathbb{R}^{n}$, there exists $R_{K} \in\left(0, \min \left\{\delta\left(X^{+}\right), \delta\left(X^{-}\right)\right\}\right)$such that for $4 r<R_{K}$ and $Q \in \partial \Omega \cap K$,

$$
\frac{\omega^{ \pm}(B(Q, r))}{r^{n-1}} \sim\left(\frac{1}{r^{2}} \int_{B(Q, r)} \frac{\left|\nabla u^{ \pm}\right|^{2}}{|X-Q|^{n-2}} d X\right)^{\frac{1}{2}}
$$

and

$$
\gamma(Q, r)^{\frac{1}{2}} \sim \frac{\omega^{+}(B(Q, r))}{r^{n-1}} \cdot \frac{\omega^{-}(B(Q, r))}{r^{n-1}} .
$$

The proof is a straightforward combination of the doubling property of $\omega^{ \pm}$(see (3.16)), (3.9), (3.14) and (3.15). The constants that appear in (3.29) and (3.30) depend on the set $K$.

We turn our attention to the tangent structure of 2-sided locally NTA domains.

Let $\Omega \subset \mathbb{R}^{n}$ be a 2-sided locally NTA domain. Let $\left\{r_{j}\right\}_{j \geq 1}$ be a sequence of positive numbers such that $\lim _{j \rightarrow \infty} r_{j}=0$. For $Q \in \partial \Omega$, consider

$$
\Omega_{j}^{ \pm}=\frac{1}{r_{j}}\left(\Omega^{ \pm}-Q\right) \text { with } \partial \Omega_{j}^{ \pm}=\frac{1}{r_{j}}\left(\partial \Omega^{ \pm}-Q\right),
$$

the functions

$$
u_{j}^{ \pm}(X)=\frac{u^{ \pm}\left(r_{j} X+Q\right)}{\omega^{ \pm}\left(B\left(Q, r_{j}\right)\right)} r_{j}^{n-2}
$$

and the measures

$$
\omega_{j}^{ \pm}(E)=\frac{\omega^{ \pm}\left(r_{j} E+Q\right)}{\omega^{ \pm}\left(B\left(Q, r_{j}\right)\right)} \text { for } E \subset \mathbb{R}^{n} \text { a Borel set. }
$$

Note that Lemma 3.4 ensures that given a compact set $K \subset \mathbb{R}^{n}$ containing $Q$, for $j$ large enough (depending only on $K$ ),

$$
C_{K}^{-1} \leq \frac{u^{ \pm}\left(A^{ \pm}\left(Q, r_{j}\right)\right)}{\omega^{ \pm}\left(B\left(Q, r_{j}\right)\right)} r_{j}^{n-2} \leq C_{K} .
$$

Here $C_{K}$ is a constant that only depends on $K$, and $A^{ \pm}\left(Q, r_{j}\right)$ denote the nontangential points associated to $Q$ at radius $r_{j}$ in $\Omega^{ \pm}$.

The boundary Harnack principle (see Lemma 3.3) yields that for $N>1, X \in$ $B(0, N)$ and $j$ large enough depending only on $N$

$$
u^{ \pm}\left(r_{j} X+Q\right) \leq C_{N, K} u^{ \pm}\left(A^{ \pm}\left(Q, r_{j}\right)\right) .
$$

Thus combining (3.32) and (3.34) we obtain that

$$
\sup _{j \geq 1} \sup _{X \in B(0, N)} u_{j}^{ \pm}(X) \leq C_{N, K}<\infty .
$$

Furthermore since $\omega^{ \pm}$are locally doubling (see Lemma 3.5),

$$
\sup _{j \geq 1} \omega_{j}^{ \pm}(B(0, N)) \leq C_{N, K}<\infty .
$$


Theorem 3.3. Let $\Omega \subset \mathbb{R}^{n}$ be a 2-sided locally NTA domain. Using the notation above, we have that there exists a sequence (which we relabel) satisfying as $j \rightarrow \infty$

$$
\begin{aligned}
\Omega_{j}^{ \pm} \rightarrow \Omega_{\infty}^{ \pm} & \text {in the Hausdorff distance sense } \\
& \text { uniformly on compact sets, } \\
\partial \Omega_{j}^{ \pm} \rightarrow \partial \Omega_{\infty}^{ \pm} & \begin{array}{l}
\text { in the Hausdorff distance sense } \\
\text { uniformly on compact sets, }
\end{array}
\end{aligned}
$$

where $\Omega_{\infty}^{ \pm}$are unbounded NTA domains with $\partial \Omega_{\infty}^{+}=\partial \Omega_{\infty}^{-}$. Moreover, there exist $u_{\infty}^{ \pm} \in C\left(\mathbb{R}^{n}\right)$ such that

$$
u_{j}^{ \pm} \rightarrow u_{\infty}^{ \pm} \text {uniformly on compact sets }
$$

and

$$
\left\{\begin{array}{cl}
\Delta u_{\infty}^{ \pm}=0 & \text { in } \Omega_{\infty}^{ \pm}, \\
u_{\infty}^{ \pm}=0 & \text { on } \partial \Omega_{\infty}^{ \pm} \\
u_{\infty}^{ \pm}>0 & \text { in } \Omega_{\infty}^{ \pm} .
\end{array}\right.
$$

Furthermore

$$
\omega_{j}^{ \pm} \rightarrow \omega_{\infty}^{ \pm} \text {weakly as Radon measures. }
$$

Here $\omega_{\infty}^{ \pm}$are the harmonic measures of $\Omega_{\infty}^{ \pm}$with pole at infinity, corresponding to $u_{\infty}^{ \pm}$, i.e. $\forall \varphi \in C_{c}^{\infty}\left(\mathbb{R}^{n}\right)$,

$$
\int_{\Omega_{\infty}^{ \pm}} u_{\infty}^{ \pm} \Delta \varphi=\int_{\partial \Omega_{\infty}^{ \pm}} \varphi d \omega_{\infty}^{ \pm}
$$

For the proof of this theorem see 14, $\S 4$.

When $\Omega$ is a 2 -sided locally NTA domain, by the differentiation theory of Radon measures (see [7]) we know that

$$
\partial \Omega=\Lambda_{1} \cup \Lambda_{2} \cup \Lambda_{3} \cup \Lambda_{4},
$$

where

$\Lambda_{1}=\left\{Q \in \partial \Omega: 0<h(Q):=\frac{d \omega^{-}}{d \omega^{+}}(Q)=D_{\omega^{+} \omega^{-}}(Q)=\lim _{r \rightarrow 0} \frac{\omega^{-}(B(Q, r))}{\omega^{+}(B(Q, r))}<\infty\right\}$,

$$
\begin{aligned}
& \Lambda_{2}=\left\{Q \in \partial \Omega: D_{\omega+} \omega^{-}(Q)=\lim _{r \rightarrow 0} \frac{\omega^{-}(B(Q, r))}{\omega^{+}(B(Q, r))}=\infty\right\} \text {, } \\
& \Lambda_{3}=\left\{Q \in \partial \Omega: D_{\omega^{+}} \omega^{-}(Q)=\lim _{r \rightarrow 0} \frac{\omega^{-}(B(Q, r))}{\omega^{+}(B(Q, r))}=0\right\}, \\
& \Lambda_{4}=\left\{Q \in \partial \Omega: \lim _{r \rightarrow 0} \frac{\omega^{-}(B(Q, r))}{\omega^{+}(B(Q, r))} \text { does not exist }\right\} .
\end{aligned}
$$

Note that:

- $\omega^{+}\left(\Lambda_{2}\right)=0, \omega^{-}\left(\Lambda_{3}\right)=0$ and $\omega^{ \pm}\left(\Lambda_{4}\right)=0$.

- $\omega^{+} \perp \omega^{-}$in $\Lambda_{2} \cup \Lambda_{3}$.

- $\omega^{+}\left\llcorner\Lambda_{1}\right.$ and $\omega^{-}\left\llcorner\Lambda_{1}\right.$ are mutually absolutely continuous.

- By the Radon-Nikodym theorem $h \in L_{l o c}^{1}\left(\omega^{+}\right)$and $\frac{1}{h} \in L_{l o c}^{1}\left(\omega^{-}\right)$. 
Define

$\Gamma=\left\{Q \in \Lambda_{1}: Q\right.$ density point of $\Lambda_{1}$ with respect to $\omega^{ \pm}$,

$$
\left.h(Q)=\lim _{r \rightarrow 0} f_{B(Q, r)} h d \omega^{+}, \lim _{r \rightarrow 0} f_{B(Q, r)}|h(P)-h(Q)| d \omega^{+}(P)=0\right\} .
$$

Note that $\omega^{ \pm}\left(\Lambda_{1} \backslash \Gamma\right)=0$.

Theorem 3.4. Let $\Omega \subset \mathbb{R}^{n}$ be a 2-sided locally NTA domain. For $Q \in \Gamma$ (defined in (3.49) ) the blow-up procedure in Theorem 3.3 yields

$$
\begin{aligned}
& \omega_{\infty}^{+}=\omega_{\infty}^{-}, \\
& u_{\infty}=u_{\infty}^{+}-u_{\infty}^{-} \text {is a harmonic polynomial in } \mathbb{R}^{n} .
\end{aligned}
$$

Furthermore there exists $\eta=\eta(n)>0$ such that if $\Omega$ is a $\eta$-Reifenberg flat domain (i.e. for each compact set $K \subset \mathbb{R}^{n}$ there exists $r_{K}>0$ so that for $P \in \partial \Omega \cap K$, and $\left.r \in\left(0, r_{K}\right), \beta_{\infty}(P, r)<\eta(n)\right)$, then $u_{\infty}$ is linear. Here

$$
\beta_{\infty}(P, r)=\frac{1}{r} \inf _{L \in G(n, n-1)} D[\partial \Omega \cap B(P, r) ; L \cap B(P, r)],
$$

and $D$ denotes the Hausdorff distance between sets.

Proof. Let $Q \in \Gamma$, and let $\left\{r_{j}\right\}_{j \geq 1}$ be a sequence of positive numbers such that $\lim _{j \rightarrow \infty} r_{j}=0$. Suppose that (3.38), (3.39), (3.40), (3.41) and (3.42) hold. Let $\varphi \in C_{c}\left(\mathbb{R}^{n}\right)$; then

$$
\int_{\partial \Omega_{j}^{ \pm}} \varphi d \omega_{j}^{ \pm}=\frac{1}{\omega^{ \pm}\left(B\left(Q, r_{j}\right)\right)} \int_{\partial \Omega^{ \pm}} \varphi\left(\frac{P-Q}{r_{j}}\right) d \omega^{+}(P) .
$$

In particular if spt $\varphi \in B(0, M)$, then

$$
\begin{aligned}
& \int_{\partial \Omega_{j}^{ \pm}} \varphi d \omega_{j}^{-}= \frac{1}{\omega^{-}\left(B\left(Q, r_{j}\right)\right)} \int_{\partial \Omega} \varphi\left(\frac{P-Q}{r_{j}}\right) h(P) d \omega^{+}(P) \\
&+\frac{1}{\omega^{-}\left(B\left(Q, r_{j}\right)\right)} \int_{\partial \Omega \cap \Lambda_{2}} \varphi\left(\frac{P-Q}{r_{j}}\right) d \omega^{-}(P)
\end{aligned}
$$

$$
\begin{aligned}
& \frac{1}{\omega^{-}\left(B\left(Q, r_{j}\right)\right)} \int_{\partial \Omega} \varphi\left(\frac{P-Q}{r_{j}}\right) h(P) d \omega^{+}(P) \\
= & \frac{\omega^{+}\left(B\left(Q, r_{j}\right)\right)}{\omega^{-}\left(B\left(Q, r_{j}\right)\right)} \cdot \frac{1}{\omega^{+}\left(B\left(Q, r_{j}\right)\right)} \int \varphi\left(\frac{P-Q}{r_{j}}\right) h(P) d \omega^{+}(P) \\
= & h(Q) \cdot \frac{\omega^{+}\left(B\left(Q, r_{j}\right)\right)}{\omega^{-}\left(B\left(Q, r_{j}\right)\right)} \cdot \frac{1}{\omega^{+}\left(B\left(Q, r_{j}\right)\right)} \int_{\partial \Omega} \varphi\left(\frac{P-Q}{r_{j}}\right) d \omega^{+}(P) \\
& +\frac{\omega^{+}\left(B\left(Q, r_{j}\right)\right)}{\omega^{-}\left(B\left(Q, r_{j}\right)\right)} \cdot \frac{1}{\omega^{+}\left(B\left(Q, r_{j}\right)\right)} \int_{\partial \Omega} \varphi\left(\frac{P-Q}{r_{j}}\right)(h(P)-h(Q)) d \omega^{+}(P) .
\end{aligned}
$$


Thus using the fact that $\omega^{ \pm}$is locally doubling, for $Q \in \Gamma$, (3.54) and (3.55) yield

$$
\begin{aligned}
& \left|\int_{\partial \Omega_{j}^{-}} \varphi d \omega_{j}^{-}-\frac{h(Q)}{f_{B\left(Q, r_{j}\right)} h d \omega^{+}} \int_{\partial \Omega_{j}^{+}} \varphi d \omega_{j}^{+}\right| \\
& \leq\|\varphi\|_{\infty} \cdot \frac{\omega^{+}\left(B\left(Q, r_{j}\right)\right)}{\omega^{-}\left(B\left(Q, r_{j}\right)\right)} \cdot \frac{\omega^{+}\left(B\left(Q, M r_{j}\right)\right)}{\omega^{+}\left(B\left(Q, r_{j}\right)\right)} f_{B\left(Q, M r_{j}\right)}|h(P)-h(Q)| d \omega^{+}(P) \\
& +\|\varphi\|_{\infty} \frac{\omega^{-}\left(B\left(Q, M r_{j}\right) \cap \Lambda_{2}\right)}{\omega^{-}\left(B\left(Q, r_{j}\right)\right)} \\
& \leq C_{K, M}\|\varphi\|_{\infty}\left\{\frac{\omega^{+}\left(B\left(Q, r_{j}\right)\right)}{\omega^{-}\left(B\left(Q, r_{j}\right)\right)}\right. \\
& \left.f_{B\left(Q, M r_{j}\right)}|h(P)-h(Q)| d \omega^{+}(P)+\frac{\omega^{-}\left(B\left(Q, M r_{j}\right) \backslash \Lambda_{1}\right)}{\omega^{-}\left(B\left(Q, M r_{j}\right)\right)}\right\} .
\end{aligned}
$$

Since $Q \in \Gamma$, by letting $j \rightarrow \infty$ we obtain

$$
\int_{\partial \Omega_{\infty}^{+}} \varphi d \omega_{\infty}^{-}=\int_{\partial \Omega_{\infty}^{-}} \varphi d \omega_{\infty}^{+}
$$

for every $\varphi \in C_{c}\left(\mathbb{R}^{n}\right)$. Since $\partial \Omega_{\infty}^{+}=\partial \Omega_{\infty}^{-}$, to show that $u_{\infty}=u_{\infty}^{+}-u_{\infty}^{-}$is harmonic in $\mathbb{R}^{n}$, let $\varphi \in C_{c}^{\infty}(\mathbb{R})$. By (3.57) we have

$$
\begin{aligned}
\int_{\mathbb{R}^{n}} u_{\infty} \Delta \varphi & =\int_{\Omega_{\infty}^{+}} u_{\infty} \Delta \varphi d Y-\int_{\Omega_{\infty}^{-}} u_{\infty} \Delta \varphi d Y \\
& =\int_{\partial \Omega_{\infty}^{+}} \varphi d \omega_{\infty}^{+}-\int_{\partial \Omega_{\infty}^{-}} \varphi d \omega_{\infty}^{-}=0 .
\end{aligned}
$$

Since $u_{\infty}$ is continuous in $\mathbb{R}^{n}$, it is weakly harmonic and therefore harmonic in $\mathbb{R}^{n}$. Note that $u_{\infty}(0)=0$. An argument similar to the one that appears in the proof of Theorem 4.4 in [14 shows that $u_{\infty}$ is a harmonic polynomial. Theorem 4.1 in [13] shows that given $\delta>0$, there exists $\eta>0$ such that if $\Omega$ is $\eta$-Reifenberg flat, then $\omega^{+}$is $\delta$-doubling as in Definition 4.4 in [14. The same argument as in the proof of Theorem 4.4 in [14] shows in this case that if $\omega^{+}$is $\delta$-doubling with $n \delta<1$, then $u_{\infty}$ is linear.

Corollary 3.1. There exists $\eta>0$ such that if $\Omega$ is a $\eta$-Reifenberg flat domain, then

$$
\operatorname{dim}_{\mathcal{H}} \Gamma \leq n-1
$$

Proof. Theorem 3.1 in 13 shows that if $\eta$ is small enough depending only on $n$, then $\Omega$ is a 2-sided locally NTA domain. Thus by Theorem 3.4 for $Q \in \Gamma$ all blowups of $\partial \Omega$ at $Q$ are the zero set of a linear polynomial that is an $(n-1)$-plane. For $Q \in \partial \Omega$, the last remark in Theorem 3.4 ensures that $\lim _{r \rightarrow 0} \beta_{\infty}(Q, r)=0$. Thus, given $\epsilon>0$, there exists $r_{Q, \epsilon}>0$ such that for $r<r_{Q, \epsilon}, \beta_{\infty}(Q, r)<\epsilon$, which implies that there exists an $(n-1)$-plane $L(Q, r)$ through $Q$ so that

$$
\partial \Omega \cap B(Q, r) \subset \partial \Omega \cap B(Q, r) \subset(L(Q, r) \cap B(Q, r) ; \epsilon r) .
$$


Thus for $Q \in \partial \Omega, \lim _{r \rightarrow 0} \beta_{\partial \Omega}(Q, r)=0$. Lemma 2.4 yields the conclusion of the corollary.

\section{TAngent Structure And SIZE of $\Gamma$}

Let $\mathcal{F}$ be the set of $(n-1)$ flat measures in $\mathbb{R}^{n}$, i.e.

$$
\mathcal{F}=\left\{c \mathcal{H}^{n-1}\llcorner V: c \in(0, \infty) ; V \in G(n, n-1)\} .\right.
$$

Note that since $G(n, n-1)$ is compact, $\mathcal{F}$ has a compact basis, and it is closed under the weak convergence of Radon measure.

Lemma 4.1. Let $h$ be a harmonic polynomial in $\mathbb{R}^{n}$ such that $h(0)=0$, and $\{h>0\}$ and $\{h<0\}$ are unbounded NTA domains. Let $\omega$ be the corresponding harmonic measure, i.e. $\forall \varphi \in C_{c}^{\infty}\left(\mathbb{R}^{n}\right)$

$$
\int_{\{h>0\}} h^{+} \Delta \varphi=\int_{\{h<0\}} h^{-} \Delta \varphi=\int_{\{h=0\}} \varphi d \omega .
$$

There exists $\epsilon_{0}>0$ (depending on the NTA constant of $\{h>0\}$ and on $n$ ) such that if for some $r_{0}>0$

$$
d_{r}(\omega, \mathcal{F})<\epsilon_{0} \quad \text { for } r \geq r_{0}, \quad \text { then } \omega \in \mathcal{F} .
$$

Remark 4.1. Note that $h$ is the Green's function with pole at infinity for $\{h>0\}$ and $\omega$ is its corresponding harmonic measure.

Proof. Let $\tau>1$ and $r \geq r_{0}$; there exists $\Psi \in \mathcal{F}$ such that $F_{\tau r}(\Psi)=1$ and

$$
F_{r}\left(\frac{\omega}{F_{\tau r}(\omega)}, \Psi\right) \leq F_{r \tau}\left(\frac{\omega}{F_{\tau r}(\omega)}, \Psi\right)<\epsilon_{0} .
$$

Thus

$$
F_{r}(\Psi)-\epsilon_{0} \leq \frac{F_{r}(\omega)}{F_{\tau r}(\omega)} \leq F_{r}(\Psi)+\epsilon_{0}
$$

Since $\Psi=c \mathcal{H}^{n-1}\left\llcorner V, F_{r \tau}(\Psi)=1=c \frac{\omega_{n-1}}{n}(\tau r)^{n}\right.$ and $F_{r}(\Psi)=\tau^{-n}$. Thus given $\delta>0$ (small enough) for $\tau \in\left(1, \tau_{\epsilon_{0}, \delta}\right)$ with $\tau_{\epsilon_{0}, \delta}=\left(\frac{\delta \epsilon_{0}^{-1}}{2}\right)^{\frac{1}{n}}$ for $r \geq r_{0}$, (4.5) yields

$$
(1+\delta)^{-1} \tau^{-n}<\frac{F_{r}(\omega)}{F_{\tau r(\omega)}}<(1+\delta) \tau^{-n} .
$$

Applying (4.6) to $\tau^{j} r$ for $j=1, \ldots, \ell$ with $\ell \in \mathbb{N}$ and $r \geq r_{0}$, then multiplying the outcomes we obtain

$$
\left[(1+\delta)^{-1} \tau^{-n}\right]^{\ell} \leq \frac{F_{r}(\omega)}{F_{\tau^{\ell} r}(\omega)} \leq\left[(1+\delta) \tau^{-n}\right]^{\ell} .
$$

Since $\omega$ is a doubling measure with the doubling constant depending only on the NTA constant of $\{h>0\}$ and on $n$ (see [15] Lemma 3.1 or [11] Lemma 4.9,4.11), from the definition of $F_{r}$ (see Definition 2.1) we have that there is $C>1$ such that for $r>0$

$$
C^{-1} r \omega(B(0, r)) \leq \frac{r}{2} \omega\left(B\left(0, \frac{r}{2}\right)\right) \leq F_{r}(\omega) \leq r \omega(B(0, r)) .
$$

Combining (4.7) and (4.8) we obtain

$$
C^{-1}\left[(1+\delta)^{-1} \tau^{-n}\right]^{\ell} \leq \frac{\omega(B(0, r))}{\tau^{\ell} \omega\left(B\left(0, \tau^{\ell} r\right)\right)} \leq C\left[(1+\delta) \tau^{-n}\right]^{\ell} .
$$


Thus

$$
C^{-1}(1+\delta)^{-\ell} \frac{\omega\left(B\left(0, \tau^{\ell} r\right)\right)}{\left(\tau^{\ell} r\right)^{n-1}} \leq \frac{\omega(B(0, r))}{r^{n-1}} \leq C(1+\delta)^{\ell} \frac{\omega\left(B\left(0, \tau^{\ell} r\right)\right)}{\left(\tau^{\ell} r\right)^{n-1}} .
$$

By Lemma 3.4 in [15] (see also Lemma 4.8 in [11]) we know that there exists $C>1$ depending only on $n$ and on the NTA constant of $\{h>0\}$ such that

$$
C^{-1} \leq \frac{\omega(B(0, r))}{r^{n-2} h(A(0, r))} \leq C .
$$

Here $A(0, r) \in\{h>0\}$ denotes a non-tangential point for 0 at radius $r>0$. Combining (4.10) and (4.11) we have

$$
C^{-1}(1+\delta)^{-\ell} \frac{h\left(A\left(0, \tau^{\ell} r\right)\right)}{\tau^{\ell} r} \leq \frac{h(A(0, r))}{r} \leq C(1+\delta)^{\ell} \frac{h\left(A\left(0, \tau^{\ell} r\right)\right)}{\tau^{\ell} r} .
$$

If $1+\delta=\tau^{\beta}$ with $\beta \in(0,1)$, then (4.12) becomes

$$
C^{-1} \tau^{-\beta \ell} \frac{h\left(A\left(0, \tau^{\ell} r\right)\right)}{\tau^{\ell} r} \leq \frac{h(A(0, r))}{r} \leq C \tau^{\beta \ell} \frac{h\left(A\left(0, \tau^{\ell} r\right)\right)}{\tau^{\ell} r} .
$$

Note that by choosing $\delta=4 \epsilon_{0}$ (with $\epsilon_{0}>0$ to still be determined), then $\tau_{\epsilon_{0}, \delta}=$ $\tau_{0}=2^{\frac{1}{n}}$ and $1+\delta=1+4 \epsilon_{0}=\tau^{\beta}$ for some $\tau \in\left(1,2^{1 / n}\right)$ and $\beta \in(0,1)$ provided $\epsilon_{0}<\frac{1}{4}\left(2^{1 / n}-1\right)$. For $s \in\left(r_{0},+\infty\right)$ there is $\ell \geq 1$ such that $\tau^{\ell-1} r_{0}<s \leq \tau^{\ell} r_{0}$. For such $s$, the boundary Harnack's inequality (for NTA domains (see Lemma 3.3 in [15], also Lemma 4.4 in [11])), combined with (4.13), yields

$$
\begin{aligned}
\frac{h(A(0, s))}{s} & \leq C \frac{h\left(A\left(0, \tau^{\ell} r_{0}\right)\right)}{\tau^{l-1} r_{0}} \leq C \tau \frac{h\left(A\left(0, \tau^{\ell} r_{0}\right)\right)}{\tau^{\ell} r_{0}} \\
& \leq C \tau \tau^{\ell \beta} \frac{h\left(A\left(0, r_{0}\right)\right)}{r_{0}} \\
& \leq C \tau^{1+\beta}\left(\frac{s}{r_{0}}\right)^{\beta} \frac{h\left(A\left(0, r_{0}\right)\right)}{r_{0}} .
\end{aligned}
$$

Since $h$ is harmonic, using its Poisson integral formula and computing its second derivatives (as in the proof of Theorem 4.4 in [14]) from (4.14), we obtain that for $X \in B(0, s)$

$$
\left|\partial_{\alpha_{1}} \partial_{\alpha_{2}} h(X)\right| \leq C \frac{h(A(0, s))}{s^{2}} \leq C\left(\tau, r_{0}\right) s^{\beta-1} \frac{h\left(A\left(0, r_{0}\right)\right)}{r_{0}} .
$$

Since $\beta<1$, by letting $s \rightarrow \infty$ we conclude that $h$ is a polynomial of degree 1 , and therefore $\omega$ is an $(n-1)$ flat measure.

We will now return to the question of the extent to which the relative behavior of the interior and exterior harmonic measures determines the size of the boundary of a domain.

Remark 4.2. Note that for $Q \in \Gamma$

$$
\operatorname{Tan}\left(\omega^{+}, Q\right)=\operatorname{Tan}\left(\omega^{-}, Q\right) .
$$

Theorem 4.1. Let $\Omega$ be a 2-sided locally NTA domain. Let $\Gamma$ be as in (3.49), and

$$
\Gamma^{*}=\left\{Q \in \Gamma: \operatorname{Tan}\left(\omega^{ \pm}, Q\right) \cap \mathcal{F} \neq \emptyset\right\} .
$$

Then for $Q \in \Gamma^{*}, \operatorname{Tan}\left(\omega^{ \pm}, Q\right) \subset \mathcal{F}$. In particular, all blow-ups of $\partial \Omega$ at $Q \in \Gamma^{*}$ are $(n-1)$-planes, and $\operatorname{dim}_{\mathcal{H}} \Gamma^{*} \leq n-1$. Furthermore $\Gamma_{0}=\Gamma \backslash \Gamma^{*}$ satisfies $\omega^{ \pm}\left(\Gamma_{0}\right)=0$. 
Proof. For $Q \in \Gamma$ the blow-up procedure described in Theorem 3.3 always yields a harmonic polynomial (see Theorem 3.4). Let $h$ be a tangent harmonic polynomial of $u$ at $Q$, with $\{h>0\},\{h<0\}$ (unbounded NTA domains) and $\nu$ the corresponding harmonic measures to $h^{ \pm}$. By 10 the zero set of $h$, i.e. $\partial\{h>0\}$, decomposes into a disjoint union of the embedded $C^{1}$ submanifold $h^{-1}\{0\} \cap\{|D h|>0\}$, together with a closed set $h^{-1}\{0\} \cap|D h|^{-1}|0|$ which is countably $(n-2)$-rectifiable. Furthermore by Lemma 2.3. spt $\nu=h^{-1}\{0\}$. For $Y \in h^{-1}\{0\} \cap\{|D h|>0\}$ and $X \in \mathbb{R}^{n}$

$$
h_{Y, r}(X)=\frac{h(r X+Y)}{r} \underset{r \rightarrow 0}{\longrightarrow}\langle D h(Y), X\rangle
$$

uniformly on compact sets. Thus $r^{-1}(\partial\{h>0\}-Y) \rightarrow\left\langle\frac{D h(Y)}{D h(Y) \mid}\right\rangle^{\perp}=V$ as $r \rightarrow 0$, in the Hausdorff distance sense, and $r^{-(n-1)} T_{Y, r}[\nu] \rightarrow|D h(Y)| \mathcal{H}^{n-1}\llcorner V$. Therefore, for $Y \in h^{-1}\{0\} \cap\{|D h|>0\}$ all non-zero tangent measures of $\nu$ at $Y$ are flat, i.e. $\operatorname{Tan}(\nu, Y) \subset \mathcal{F}$. By Theorem 2.3 for $\omega=\omega^{ \pm}$a.e. $Q \in \Gamma$, if $\nu \in \operatorname{Tan}(\omega, Q)$, then for all $Y \in \operatorname{spt} \nu$, $\operatorname{Tan}(\nu, Y) \subset \operatorname{Tan}(\omega, Q)$. Thus, for $\omega$ a.e. $Q \in \Gamma, \mathcal{F} \cap$ $\operatorname{Tan}(\omega, Q) \neq \emptyset$, which proves that $\omega^{ \pm}\left(\Gamma_{0}\right)=0$. Our goal is to use Corollary 2.1 combined with Lemma 4.1 to show that for $Q \in \Gamma^{*}, \operatorname{Tan}(\omega, Q) \subset \mathcal{F}$. Let $\mathcal{M}=\mathcal{F} \cup \operatorname{Tan}(\omega, Q)$. Recall that $\mathcal{F}$, the set of all $(n-1)$ flat measures, is a $d$-cone with compact basis. Since $\omega$ is a doubling Radon measure, Theorem 2.2 ensures that for $Q \in \Gamma$, $\operatorname{Tan}(\omega, Q)$ is a $d$-cone with compact basis. Hence $\mathcal{M}$ is also a $d$-cone with compact basis. Moreover $\mathcal{F} \subset \mathcal{M}$, and $\mathcal{F}$ is relatively closed with respect to the weak convergence of Radon measures. By Lemma 4.1 there exists $\epsilon_{0}>0$ such that if $d_{r}(\mu, \mathcal{F})<\epsilon_{0}$ for all $r \geq r_{0}$, then $\mu \in \mathcal{F}$. Corollary 2.1 then ensures that for $Q \in \Gamma^{*}$, Tan $(\omega, Q) \subset \mathcal{F}$. Lemma 2.3 guarantees that all blow-ups of $\partial \Omega$ at $Q$ converge in the Hausdorff distance sense to an $(n-1)$-plane. Thus for $Q \in \Gamma^{*}$, $\lim _{r \rightarrow \infty} \beta_{\infty}(Q, r)=0$. As in the proof of Corollary 3.1 this implies that for $Q \in \Gamma^{*}$, $\lim _{r \rightarrow 0} \beta_{\Gamma}^{*}(Q, r)=0$. By Lemma 2.4 we conclude that $\operatorname{dim}_{\mathcal{H}} \Gamma^{*} \leq n-1$.

Corollary 4.1. Let $\Omega$ be a 2-sided locally NTA domain. Then the boundary of $\Omega$ can be decomposed as follows:

$$
\omega^{+}\left\llcorner\Gamma ^ { * } \ll \omega ^ { - } \left\llcorner\Gamma ^ { * } \ll \omega ^ { + } \left\llcorner\Gamma^{*}, \omega^{+} \perp \omega^{-} \text {in } S \text {, and } \omega^{+}(N)=\omega^{-}(N)=0\right.\right.\right. \text {. }
$$

Moreover

$$
\operatorname{dim}_{\mathcal{H}} \Gamma^{*} \leq n-1 .
$$

Furthermore, if $\omega^{ \pm}\left(\Gamma^{*}\right)>0$, then

$$
\operatorname{dim}_{\mathcal{H}} \Gamma^{*}=n-1 .
$$

Here $\Gamma^{*}$ is as in Theorem 4.1, $S=\Lambda_{2} \cup \Lambda_{3}$ (see (3.46) and (3.47)), and $N=$ $\Lambda_{1} \backslash \Gamma^{*} \cup \Lambda_{4}$.

Proof. We only need to show that (4.22) holds whenever $\omega^{ \pm}\left(\Gamma^{*}\right)>0$. By (3.12) for $Q_{0} \in \partial \Omega, r_{0}<1 / 8 \min \left\{\delta\left(X^{+}\right), \delta\left(X^{-}\right)\right\}, Q \in \Gamma^{*} \cap B\left(Q_{0}, \frac{r_{0}}{2}\right)$ and $0<r<r_{0}$,

$$
\begin{aligned}
\frac{\omega^{+}(B(Q, r))}{r^{n-1}} \cdot \frac{\omega^{-}(B(Q, r))}{r^{n-1}} & \leq C\left(Q_{0}, r_{0}\right), \\
\left(f_{B(Q, r)} h d \omega^{+}\right)\left(\frac{\omega^{+}(B(Q, r))}{r^{n-1}}\right)^{2} & \leq C\left(Q_{0}, r_{0}\right) .
\end{aligned}
$$


Thus

$$
\frac{\log \left(f_{B(Q, r)} h d \omega^{+}\right)^{1 / 2}}{\log r}+\frac{\log \omega^{+}(B(Q, r))}{\log r} \geq n-1+\frac{\log C\left(Q_{0}, r_{0}\right)}{\log r} .
$$

Letting $r$ tend to 0 in (4.24) we obtain that

$$
\liminf _{r \rightarrow 0} \frac{\log \omega^{+}(B(Q, r))}{\log r} \geq n-1 .
$$

By Proposition 2.3 in 8 from (4.25) we conclude that, since $\omega^{+}\left(\Gamma^{*}\right)>0, \operatorname{dim}_{\mathcal{H}} \Gamma^{*} \geq$ $n-1$.

Theorem 4.2. Let $\Omega$ be a 2-sided locally NTA domain such that $\mathcal{H}^{n-1}\llcorner\partial \Omega$ is a Radon measure. Then as in Theorem 4.1, $\partial \Omega=\Gamma^{*} \cup S \cup N$ and $\Gamma^{*}$ is $(n-1)$ rectifiable.

Proof. Our strategy consists in proving that the density of $\mathcal{H}^{n-1}\left\llcorner\Gamma^{*}\right.$ exists and is 1 a.e. Then we appeal to Theorem 17.6 in [18, which provides a rectifiability criteria.

First we prove that for $Q \in \Gamma^{*}$ (see (4.17) for the definition)

$$
\Theta_{*}^{n-1}\left(\mathcal{H}^{n-1}\llcorner\partial \Omega, Q)=\liminf _{r \rightarrow 0} \frac{\mathcal{H}^{n-1}(B(Q, r) \cap \partial \Omega)}{\omega_{n-1} r^{n-1}} \geq 1 .\right.
$$

For $Q \in \Gamma^{*}$ and $\delta>0$ by Theorem 4.1 there exists $r_{0}>0$ so that for $r<r_{0}$ there exists $L(Q, r)$ an $(n-1)$-plane containing $Q$ so that

$$
\frac{1}{r} D[\partial \Omega \cap B(Q, r) ; L(Q, r) \cap B(Q, r)] \leq \delta .
$$

Since $\Omega^{ \pm}$satisfies the corkscrew condition, we may assume that for $r<r_{0}$ there exist $A^{ \pm}(Q, r) \subset \Omega^{ \pm}$so that

$$
B\left(A^{ \pm}(Q, r), \frac{r}{M}\right) \subset \Omega^{ \pm} \cap B(Q, r) .
$$

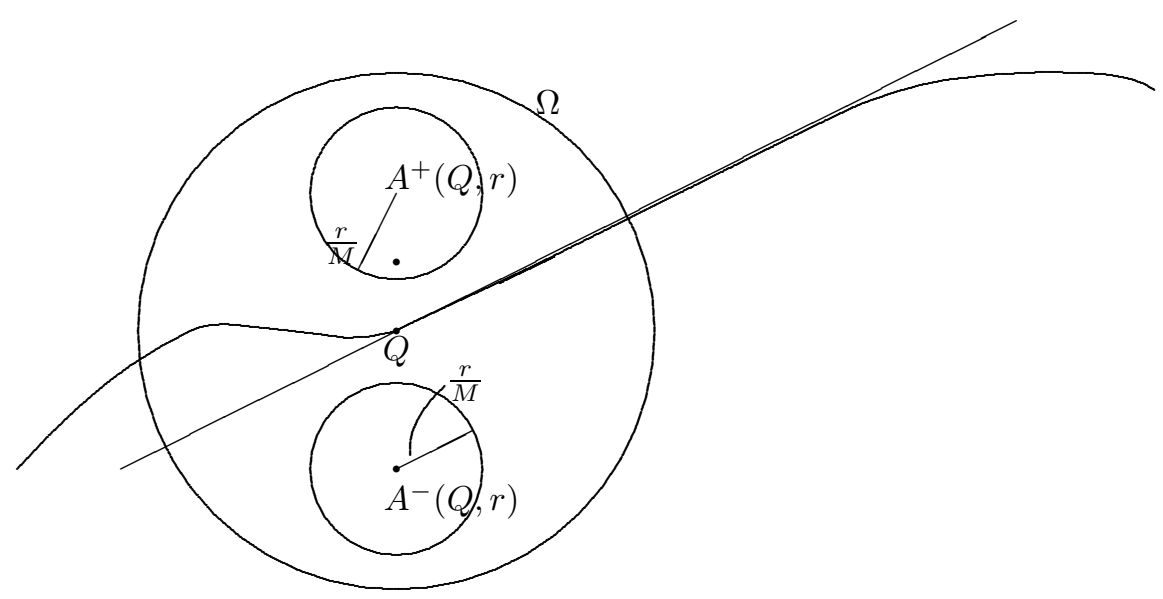


If $\vec{n}(Q, r)$ denotes the unit normal to $L(Q, r)$, (4.27) and (4.28) ensure that for $\delta$ small $\left(\delta<\frac{1}{2 M}\right)$

$$
\left|\left\langle A^{ \pm}(Q, r)-Q, \vec{n}(Q, r)\right\rangle\right| \geq 2 \delta r .
$$

We may assume that $\left\langle A^{+}(Q, r)-Q, \vec{n}(Q, r)\right\rangle \geq 2 \delta r$. If $Z \in B(Q, r)$ and $\langle Z-$ $Q, \vec{n}(Q, r)\rangle \geq 2 \delta r$, then $Z \in \Omega^{+}$. Otherwise $Z \in \Omega^{-}$(since $Z \notin \partial \Omega$ by (4.27)), and by connectivity there would be a point $P \in \partial \Omega$ in the segment joining $A^{+}(Q, r)$ to $Z$. Such $P$ would satisfy $\langle P-Q, \vec{n}(Q, r)\rangle \geq 2 \delta r$ which contradicts (4.27). This proves that

$$
\{Z \in B(Q, r):\langle Z-Q, \vec{n}(Q, r)\rangle \geq 2 \delta r\} \subset \Omega^{+} \cap B(Q, r)
$$

and

$$
\{Z \in B(Q, r):\langle Z-Q ; \vec{n}(Q, r)\rangle \leq-2 \delta r\} \subset \Omega^{-} \cap B(Q, r) .
$$

Thus for $x \in L(Q, r) \cap B\left(Q, r \sqrt{1-4 \delta^{2}}\right)$ a simple connectivity argument shows that there exists $P \in \partial \Omega$ such that $P=(x, t)$ with $|t|<\delta r$. Hence $P \in \partial \Omega \cap B(Q, r)$. If $\pi_{Q, r}$ denotes the orthogonal projection onto $L(Q, r)$ we have for $\delta$ small enough

$$
\begin{aligned}
\mathcal{H}^{n-1}(\partial \Omega \cap B(Q, r)) & \geq \mathcal{H}^{n-1}\left(\Pi_{Q, r}(\partial \Omega \cap B(Q, r))\right. \\
& \geq \omega_{n-1} r^{n-1}\left(1-4 \delta^{2}\right)^{\frac{n-1}{2}} \\
& \geq \omega_{n-1} r^{n-1}(1-\delta),
\end{aligned}
$$

which ensures that (4.26) holds. Since $\mathcal{H}^{n-1}\left\llcorner\partial \Omega\right.$ is a Radon measure for $\mathcal{H}^{n-1}\llcorner\partial \Omega$ a.e. $Q$,

$$
\Theta^{*, n-1}\left(\mathcal{H}^{n-1}\llcorner\partial \Omega, Q)=\limsup _{r \rightarrow 0} \frac{\mathcal{H}^{n-1}(B(Q, r) \cap \partial \Omega)}{\omega_{n-1} r^{n-1}} \leq 1 ;\right.
$$

see [18] in Theorem 6.2. Thus combining (4.26) and (4.32) we conclude that for $Q \in \Gamma^{*}$,

$$
\Theta^{n-1}\left(\mathcal{H}^{n-1}\llcorner\partial \Omega, Q)=\lim _{r \rightarrow 0} \frac{\mathcal{H}^{n-1}(B(Q, r) \cap \partial \Omega)}{\omega_{n-1} r^{n-1}}=1 .\right.
$$

Thus since $\mathcal{H}^{n-1}\left\llcorner\partial \Omega\right.$ is a Radon measure, by Corollary 6.3 in 18 for $\mathcal{H}^{n-1}$ a.e. $Q \in \Gamma^{*}$,

$$
\Theta^{n-1}\left(\mathcal{H}^{n-1}\llcorner\partial \Omega, Q)=\Theta^{n-1}\left(\mathcal{H}^{n-1}\left\llcorner\Gamma^{*}, Q\right)=1 .\right.\right.
$$

Therefore Theorem 17.6 in 18 ensures that $\Gamma^{*}$ is $(n-1)$-rectifiable.

The following theorem proves that there are no Wolff snowflakes for which $\omega^{+}$ and $\omega^{-}$are mutually absolutely continuous, answering a question in [16].

Theorem 4.3. Let $\Omega$ be a 2-sided locally NTA domain. Assume that $\omega^{+}$and $\omega^{-}$ are mutually absolutely continuous; then

$$
\mathcal{H}-\operatorname{dim} \omega^{+}=\mathcal{H}-\operatorname{dim} \omega^{-}=n-1 .
$$

Here the Hausdorff dimension of $\omega^{ \pm}, \mathcal{H}-\operatorname{dim} \omega^{ \pm}$is defined as in (1.2).

Proof. Since $\omega^{+}$and $\omega^{-}$are mutually absolutely continuous, it is easy to see that $\mathcal{H}-\operatorname{dim} \omega^{+}=\mathcal{H}-\operatorname{dim} \omega^{-}$. For each compact set $K \subset \mathbb{R}^{n}, \omega^{ \pm}(\Gamma \cap K)=\omega^{ \pm}(\partial \Omega \cap K)$. Hence for $\Gamma^{*}=\Gamma \backslash \Gamma_{0}$ with $\Gamma_{0}$ as in Theorem 4.1] $\omega^{ \pm}\left(\Gamma^{*} \cap K\right)=\omega^{ \pm}(\partial \Omega \cap K)$ and $\operatorname{dim}_{\mathcal{H}} \Gamma^{*} \leq n-1$, i.e. $\forall \kappa>n-1, \mathcal{H}^{\kappa}\left(\Gamma^{*}\right)=0$, which implies that $\mathcal{H}-\operatorname{dim} \omega^{+} \leq n-1$ and $\mathcal{H}-\operatorname{dim} \omega^{-} \leq n-1$. Since in this case $\omega^{ \pm}\left(\Gamma^{*}\right)>0$ (by (4.19) and (4.20), (4.22) yields (4.35). 
We conclude by having a second look at $\Gamma$ motivated by the 2-dimensional results in Chapter VI of [9]. Denote it by $\omega=\omega^{ \pm}$, and define

$$
\begin{aligned}
& \Gamma_{g}=\left\{Q \in \Gamma: 0<\limsup _{r \rightarrow 0} \frac{\omega(B(Q, r))}{r^{n-1}}<\infty\right\}, \quad \Gamma_{g}^{*}=\Gamma_{g} \cap \Gamma^{*}, \\
& \Gamma_{b}=\left\{Q \in \Gamma: \limsup _{r \rightarrow 0} \frac{\omega(B(Q, r))}{r^{n-1}}=0\right\} \quad \Gamma_{b}^{*}=\Gamma_{b} \cap \Gamma^{*} .
\end{aligned}
$$

Since $\omega^{+}\left\llcorner\Lambda_{1}\right.$ and $\omega^{-}\left\llcorner\Lambda_{1}\right.$ are mutually absolutely continuous, and $\Gamma \subset \Lambda_{1}$ (see (3.49) $), \Gamma_{g}$ and $\Gamma_{b}$ are well defined.

By (3.12) for $Q_{0} \in \partial \Omega, r_{0}<1 / 8 \min \left\{\delta\left(X^{+}\right), \delta\left(X^{-}\right)\right\}, Q \in \Gamma \cap B\left(Q_{0}, \frac{r_{0}}{2}\right)$ and $0<r<r_{0}$,

$$
\left(f_{B(Q, r)} h d \omega^{+}\right)\left(\frac{\omega^{+}(B(Q, r))}{r^{n-1}}\right)^{2} \leq C\left(Q_{0}, r_{0}\right)
$$

Thus for $Q \in \Gamma, \lim \sup _{r \rightarrow 0} \frac{\omega(B(Q, r))}{r^{n-1}}<\infty$, which ensures that $\Gamma=\Gamma_{g} \cup \Gamma_{b}$.

Lemma 4.2. Let $\Omega$ be a 2 -sided locally NTA domain. Then $\mathcal{H}^{n-1}\left\llcorner\Gamma_{g}\right.$ and $\omega\left\llcorner\Gamma_{g}\right.$ are mutually absolutely continuous. In particular $\mathcal{H}^{n-1}\left\llcorner\Gamma_{g}\right.$ is $\sigma$-finite. Furthermore $\Gamma^{*}=\Gamma_{g}^{*} \cup \Gamma_{b}^{*} \cup Z$ with $\omega(Z)=0$. Moreover if for $E \subset \mathbb{R}^{n}$ Borel, $\omega\left(\Gamma_{b}^{*} \cap E\right)>0$, then $\mathcal{H}^{n-1}\left(\Gamma_{b}^{*} \cap E\right)=\infty$.

Proof. Let

$$
\Gamma_{g}=\bigcup_{i=1}^{\infty} \Gamma_{g}^{i}=\bigcup_{i=1}^{\infty}\left\{Q \in \Gamma_{g} ; 2^{-i} \leq \limsup _{r \rightarrow 0} \frac{\omega(B(Q, r))}{r^{n-1}} \leq 2^{i}\right\} .
$$

By Proposition 2.2 in [8] for any Borel set $E \subset \Gamma$ and $i, k \in \mathbb{N}$,

$$
2^{-i} \omega\left(E \cap \Gamma_{g}^{i} \cap B(0, k)\right) \leq \mathcal{H}^{n-1}\left(E \cap \Gamma_{g}^{i} \cap B(0, k)\right) \leq 2^{n+i} \omega\left(E \cap \Gamma_{g}^{i} \cap B(0, k)\right),
$$

which proves the statements that $\mathcal{H}^{n-1}\left\llcorner\Gamma_{g}\right.$ and $\omega\left\llcorner\Gamma_{g}\right.$ are mutually absolutely continuous. The statement about $\Gamma_{b}$ is a simple consequence of Proposition 2.2 in [8].

Corollary 4.2. Let $\Omega$ be a 2-sided locally NTA domain. Then the boundary of $\Omega$ can be decomposed as follows:

$$
\partial \Omega=\Gamma_{g}^{*} \cup \Gamma_{b}^{*} \cup S \cup \widetilde{N},
$$

where

On $\Gamma_{g}^{*}, \mathcal{H}^{n-1}$ is $\sigma$-finite, and $\omega$ and $\mathcal{H}^{n-1}$ are mutually absolutely continuous. On $\Gamma_{b}^{*}$ for any Borel set $E$, if $\omega\left(\Gamma_{b}^{*} \cap E\right)>0$, then $\mathcal{H}^{n-1}\left(\Gamma_{b}^{*} \cap E\right)=\infty$.

\section{ACKNOWLEDGMENT}

We are grateful to J. Garnett for his detailed explanation of the 2-dimensional results mentioned here. 


\section{REFERENCES}

1. H. W. Alt, L. A. Caffarelli \& A. Friedman, Variational problems with two phases and their free boundaries, Trans. Amer. Math. Soc. 282 (1984), 431-461. MR732100(85h:49014)

2. W. Beckner, C. Kenig \& J. Pipher, unpublished manuscript.

3. C. Bishop, Some questions concerning harmonic measure, in Partial Differential Equations with Minimal Smoothness and Applications, The IMA Volumes in Mathematics and its Applications, Volume 42, 89-98, 1992, edited by Dahlberg, Fabes, R. Fefferman, Jerison, Kenig and Pipher. MR:1155854 (93f:30023)

4. C. Bishop, L. Carleson, J. Garnett \& P. Jones, Harmonic measure supported on curves, Pacific J. Math. 138 (1989), 233-236. MR996199 (90d:30069)

5. J. Brothers \& W. Ziemer, Minimal arrangements of Sobolev functions, J. Reine Angew. Math. 384 (1988), 153-179. MR929981 (89g:26013)

6. L. Carleson, On the support of harmonic functions on sets of Cantor type, Ann. Acad. Sci. Fenn. 10 (1985), 113-123. MR802473 (87b:31002)

7. L. C. Evans \& R. F. Gariepy, Measure Theory and Fine Properties of Functions, Studies in Advanced Mathematics, CRC Press, 1992. MR.1158660 (93f:28001)

8. K. Falconer, Techniques in Fractal Geometry, Wiley \& Sons, 1997. MR1449135 (99f:28013)

9. J. Garnett \& D. Marshall, Harmonic Measure, New Mathematical Monographs:2, Cambridge University Press, 2005. MR2150803 (2006g:31002)

10. R. Hardt \& L. Simon, Nodal sets for solutions of elliptic equations, J. Differential Geom. 30 (1989), 505-522. MR:1010169 (90m:58031)

11. D. Jerison \& C. Kenig, Boundary behavior of harmonic functions in nontangentially accessible domains, Adv. in Math. 46 (1982), 80-147. MR676988(84d:31005b)

12. P. Jones \& T. Wolff, Hausdorff dimension of harmonic measure in the plane, Acta Math. 161 (1988), 131-144. MR962097(90j:31001)

13. C. Kenig \& T. Toro, Harmonic measure on locally flat domains, Duke Math. Journal 87 (1997), 509-551. MR:1446617 (98k:31010)

14. C. Kenig \& T. Toro, Free boundary regularity below the continuous threshold: 2-phase problems, J. Reine Angew. Math. 596 (2006), 1-44. MR.2254803 (2007k:35526)

15. C. Kenig \& T. Toro, Free boundary regularity for harmonic measures and Poisson kernels, Ann. of Math. 150 (1999), 369-454. MR1726699 (2001d:31004)

16. J. Lewis, G. C. Verchota, \& A. Vogel, On Wolff Snowflakes, Pacific J. of Math. 218 (2005), 139-166. MR2224593 (2006m:31005)

17. N. Makarov, Distortion of boundary sets under conformal mappings, Proc. London Math. Soc. 51 (1985), 369-384. MR794117 (87d:30012)

18. P. Mattila, Geometry of Sets and Measures in Euclidean Spaces, Cambridge University Press, 1995. MR 1333890 (96h:28006)

19. D. Preiss, Geometry of measures in $\mathbb{R}^{n}$ : distribution, rectifiability, and densities, Ann. of Math. 125 (1987), 537-643. MR890162 (88d:28008)

20. L Simon, Lectures on geometric measure theory, Australian National University, 1983. MR756417 (87a:49001)

21. T. Wolff, Counterexamples with harmonic gradients in $\mathbb{R}^{3}$, Essays in honor of Eias M. Stein, Princeton Mathematical Series 42 (1995), 321-384. MR1315554 (95m:31010)

Department of Mathematics, University of Chicago, Chicago, Illinois 60637

E-mail address: cek@math.uchicago.edu

Mathematics Institut, University of Warwick, Coventry CV4 7AL, United Kingdom E-mail address: d.preiss@warwick.ac.uk

Department of Mathematics, University of Washington, Seattle, Washington 981954350.

E-mail address: toro@math.washington.edu 